\title{
Profilin 1 delivery tunes cytoskeletal dynamics toward CNS axon regeneration
}

\author{
Rita Pinto-Costa, ${ }^{1,2}$ Sara C. Sousa, ${ }^{1,2}$ Sérgio C. Leite, ${ }^{1}$ Joana Nogueira-Rodrigues, ${ }^{1,2}$ Tiago Ferreira da Silva, ${ }^{3}$ Diana Machado, ${ }^{1}$ \\ Joana Marques, ${ }^{1}$ Ana Catarina Costa, ${ }^{1}$ Márcia A. Liz, ${ }^{1}$ Francesca Bartolini, ${ }^{4}$ Pedro Brites, ${ }^{3}$ Mercedes Costell, ${ }^{5}$ \\ Reinhard Fässler, ${ }^{6}$ and Mónica M. Sousa ${ }^{1}$ \\ ${ }^{1}$ Nerve Regeneration Group, Program in Neurobiology and Neurologic Disorders, Instituto de Biologia Molecular e Celular (IBMC) and Instituto de Inovação e Investigação em Saúde, and ${ }^{2}$ Craduate \\ Program in Molecular and Cell Biology, Instituto de Ciências Biomédicas Abel Salazar (ICBAS), Universidade do Porto, Porto, Portugal. ${ }^{3}$ NeuroLipid Biology Group, Program in Neurobiology and Neurologic \\ Disorders, Instituto de Biologia Molecular e Celular (IBMC) and Instituto de Inovação e Investigação em Saúde, Universidade do Porto, Porto, Portugal. Department of Pathology and Cell Biology, Columbia \\ University, New York, New York, USA. ${ }^{5}$ Department of Biochemistry and Molecular Biology and Estructura de Reserca Interdisciplinar en Biotecnologia i Biomedicina, Universitat de València, Valencia, Spain. \\ ${ }^{6}$ Department of Molecular Medicine, Max Plank Institute of Biochemistry, Martinsried, Germany.
}

\begin{abstract}
After trauma, regeneration of adult CNS axons is abortive, causing devastating neurologic deficits. Despite progress in rehabilitative care, there is no effective treatment that stimulates axonal growth following injury. Using models with different regenerative capacities, followed by gain- and loss-of-function analysis, we identified profilin 1 (Pfn1) as a coordinator of actin and microtubules (MTs), powering axonal growth and regeneration. In growth cones, Pfn1 increased actin retrograde flow, MT growth speed, and invasion of filopodia by MTs, orchestrating cytoskeletal dynamics toward axonal growth. In vitro, active Pfn1 promoted MT growth in a formin-dependent manner, whereas localization of MTs to growth cone filopodia was facilitated by direct MT binding and interaction with formins. In vivo, Pfn1 ablation limited regeneration of growth-competent axons after sciatic nerve and spinal cord injury. Adeno-associated viral (AAV) delivery of constitutively active Pfn1 to rodents promoted axonal regeneration, neuromuscular junction maturation, and functional recovery of injured sciatic nerves, and increased the ability of regenerating axons to penetrate the inhibitory spinal cord glial scar. Thus, we identify Pfn1 as an important regulator of axonal regeneration and suggest that AAV-mediated delivery of constitutively active Pfn1, together with the identification of modulators of Pfn1 activity, should be considered to treat the injured nervous system.
\end{abstract}

\section{Introduction}

In the adult CNS, developmental axonal growth capacity declines such that regeneration after injury is abortive. This derives from the highly inhibitory environment formed at the injury site, and the inability of CNS neurons to activate a cell-intrinsic pro-regenerative program (1). However, it is possible to stimulate the intrinsic growth capacity of specific CNS axons. In sensory dorsal root ganglia (DRG) neurons, which bear 2 axonal branches with different structure and function (2), upon injury of the peripheral axon (conditioning lesion, CL), the central axon gains growth competence and regenerates within the inhibitory CNS milieu (3). Using this model, several regeneration-associated genes and transcription factors that promote axonal regrowth were unveiled (1). In recent years, cytoskeletal organization and dynamics, especially involving actin and microtubules (MTs), have emerged as key players in axonal growth and regeneration (4). In particular, cytoskeleton modulation at the axonal tip can power the formation of a competent growth cone from a dystrophic growth-incompetent retraction bulb, promoting regeneration of CNS axons (4).

Conflict of interest: The authors have declared that no conflict of interest exists. Copyright: () 2020, American Society for Clinical Investigation.

Submitted: October 25, 2018; Accepted: January 14, 2020; Published: March 16, 2020.

Reference information: J Clin Invest. 2020;130(4):2024-2040.

https://doi.org/10.1172/JCI125771.
The peripheral domain of the growth cone is highly enriched in actin (5), a multifunctional cytoskeletal component regulated by numerous actin-binding proteins. Actin is present as either a free globular monomer - G-actin - or as part of a filament - F-actin both of which are essential for its various functions. Cyclic polymerization and depolymerization of actin filaments in the growth cone is needed to generate the mechanical force that prompts axonal elongation $(6,7)$. Local actin instability specifies neuronal polarization and axon formation. Consistently, actin-depolymerizing drugs and Rho inactivators that act on the actin cytoskeleton generate neurons with multiple axons (6). RhoA signaling is a central mediator of inhibitory cascades that hinder axonal regeneration (8-10). In this context, RhoA inhibitors improve axonal regeneration (11, 12) and are currently used in clinical trials aimed at treating spinal cord injury (SCI) (13). Nevertheless, the interplay between different actin-binding proteins controlling actin dynamics in the growth cone is still not well understood. The actin-binding and -severing protein cofilin 1 (Cfl1) (14), for example, is essential for actin remodeling during neurite formation (15). Cfl synergizes with the G-actin-binding and actin polymerization-promoting protein profilin (Pfn) to further enhance the rate of actin filament treadmilling (16). Although Cfl has been shown to be involved in powering axonal extension (17), growth cone turning during axonal pathfinding (18), and axonal regeneration (19), the role of Pfn in mammalian axonal growth has been less explored. 
In mammals, the Pfn family consists of the ubiquitously expressed Pfn1, the brain-specific Pfn2, and the testis-specific Pfn3 and Pfn4. Globally, Pfns act as nucleation/polymerizationinhibiting G-actin-sequestering molecules (20), which turn into elongators through interaction with either Ena/VASP or formins $(21,22)$. Although Pfn1 and Pfn2 are expressed in the brain, their specific role in neurons needs to be further explored. Whereas actin polymerization in neurons may be mainly regulated by Pfn1, neuronal Pfn2 seems to be specifically associated with synaptic plasticity (23). In addition to their role as regulators of actin dynamics, Pfns may also influence MT organization (24-26). Mutations in Pfn1 have been associated with neurodegenerative diseases, including amyotrophic lateral sclerosis (ALS), supporting Pfn1 relevance in neuron architectural biology. Apart from binding actin, Pfns also interact with poly-proline stretches in proteins (which are present in a vast majority of actin-binding proteins) (27), and with phosphatidylinositol 4,5-bisphosphate ( $\mathrm{PIP}_{2}$ ) (28), which links Pfn to the plasma membrane. Given the heterogeneous nature of Pfn ligands, Pfns participate in several biological processes, acting as intracellular multifunctional platforms.

Here we unveil Pfn1 as a pro-regenerative molecule that promotes actin and MT cytoskeleton crosstalk in actively growing axon terminals. Our results identify Pfn1 as a central regulator of axonal growth and regeneration and suggest new therapeutic strategies to promote axonal regrowth, specifically by interfering with Pfn1 levels and activity.

\section{Results}

Pfn1 activity increases after CL. Given the robustness of a CLin axonal growth and regeneration (3), and the importance of actin dynamics in the growth cone for axonal elongation (6), we determined how CL influences actin dynamics. For this purpose, we analyzed adult DRG neurons under 2 distinct growth modes (29): naive and regenerative growth (i.e., the growth mode resulting from a previous CL). The sciatic nerve (containing peripheral branches of DRG neurons) was lesioned in vivo (Figure 1A), and DRG neurons were collected 1 week later for in vitro culture. In cultured DRG neurons, CL increased actin dynamics in the growth cone, promoting actin retrograde flow (Figure 1, B-D), similarly to recent observations (19). In addition, CL growth cones showed increased area (Figure 1, B and E) and displayed a substantial accumulation of Pfn1 (Figure 1, F and G), raising the possibility that this protein might be important for actin dynamics in the axonal tip, and for growth competence. Next, we investigated the regulation of Pfn1 in vivo by comparing its levels following both SCI (a nonregenerative condition) and CL (a highly regenerative condition) (Figure 1A). The levels of Pfn1 were increased in DRG after CL, supporting a global increase in expression (Figure 1, H and I). Moreover, the total levels of Pfn 1 increased 7-fold at the injury site of rats with CL versus SCI, suggesting that it accumulates distally in growth cones (Figure 1, $\mathrm{J}$ and $\mathrm{K}$ ). Given that glial or myeloid cells might contribute to the effect observed in spinal cord extracts, the specific upregulation of Pfn1 in axons was assessed by immunofluorescence. In animals with CL, Pfn1 was specifically detected in the spinal cord in growth cones labeled with SCG10, a stathmin preferentially expressed in regenerating sensory axons (ref. 30 and Figure 1L), in accordance with our in vitro findings in growth cones of conditioned neurons
(Figure 1, F and G). Nonphosphorylated Pfn1 is the active form of the protein; its activity can be downregulated by RhoA/ROCKmediated phosphorylation at serine 138 (31). Importantly, in addition to increased Pfn1 levels, CL induced an 8.6-fold downregulation of the serine 138 phosphorylation of Pfn1 (Figure 1, J and K), thus increasing levels of the Pfn1 active form. In line with this finding, ROCK1, a central axon growth inhibitory molecule (32), was also 2.5-fold decreased following CL (Figure 1, J and K). Importantly, the levels of Pfn 2 were not altered by CL (Figure 1, J and K), suggesting a Pfn1-specific effect. Collectively, these data support the idea that Pfn1 is an important pro-regenerative regulator of actin dynamics in the growth cone.

Pfn1 downregulation impairs axonal growth in different neuronal types and developmental stages. To test the hypothesis that Pfn1 levels promote axonal growth, we silenced Pfn1 in cultured adult DRG neurons under naive (Figure 2, A and B) and regenerative growth (i.e., following a previous CL) conditions (Figure 2, $\mathrm{C}$ and D). In naive DRG neurons, Pfn1 knockdown (>80\% efficiency both in DRG and hippocampal neuron cultures) led to a $30 \%$ reduction in neurite length and to reduced branching when compared with DRG neurons nucleofected with an empty control plasmid (Ctrl) (Figure 2, E and F). The specificity of these effects was confirmed by expressing a human shRNA-resistant version of WT hPfn1 (WT hPfn1*), which reverted the analyzed parameters to normal levels (Figure 2, E and F). Downregulation of Pfn1 had an even more pronounced effect in conditioned DRG neurons, i.e., after activating the regenerative growth mode (CL), reducing neurite elongation by $44 \%$ (Figure $2, \mathrm{C}-\mathrm{F}$ ). Together, our results suggest that Pfn 1 is a key mediator of growth after CL. To extend our findings to additional neuronal types, we silenced Pfn1 in hippocampal neurons. When lentivirus-mediated delivery of shRNA against Pfn1 was performed, the majority of hippocampal neurons were arrested in stage 1, lacking neurite-like processes (Figure 2, $\mathrm{G}$ and $\mathrm{H})$. When shRNA plasmids were delivered through nucleofection to DIVO hippocampal neurons, neuronal polarization was delayed, resulting in an increase of 2.3- and 1.4-fold of stage 1 and stage 2 neurons, respectively (Figure 2, I and J). Similarly to naive DRG neurons, hippocampal neurons that were able to polarize had an approximately $24 \%$ reduction in axonal length (Figure $2 \mathrm{~K}$ ) and dendritic growth was reduced by over $27 \%$ (Figure $2 \mathrm{~L}$ ).

In vivo depletion of Pfn1 curbs axonal regeneration in the peripheral and central nervous systems. To determine if our in vitro findings can be extended to an in vivo system, we developed an inducible neuron-specific Pfn1-knockout mouse model using Cre-loxP technology. In this model, the yellow fluorescent protein (YFP) is coexpressed with inducible-CreER ${ }^{\mathrm{T} 2}$ (Figure $3 \mathrm{~A}$ ) and a high percentage of DRG axons in cre ${ }^{+}$Pfn1 sciatic nerves are YFP positive (Supplemental Figure 1A; supplemental material available online with this article; https://doi.org/10.1172/JCI125771DS1). Pfn1 levels were severely decreased in brain samples of $\mathrm{cre}^{+} \mathrm{Pfn} 1^{\mathrm{f} / \mathrm{l} l}$ mice when compared with $\mathrm{cre}^{+} \mathrm{Pfn} 1^{\mathrm{wt} / \mathrm{wt}}$ controls, whereas levels of $\mathrm{Pfn} 2$ remained normal (Supplemental Figure 1, B-D). Importantly, and in line with the in vitro acute shRNA-mediated knockdown, the permanent absence of Pfn1 from naive DRG neurons of adult $\mathrm{cre}^{+} \mathrm{Pfn} 1^{\mathrm{f} / \mathrm{fl}}$ mice significantly impaired neurite length (55\% reduction) and branching (Figure 3, B-D). Given the structural and possible functional similarities between the ubiquitous Pfn1 and brain-specific Pfn2, 
A

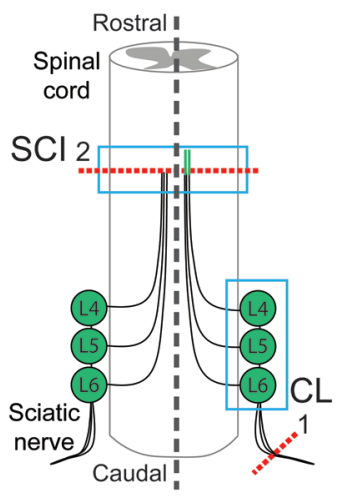

B

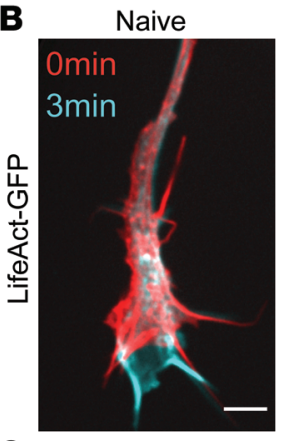

C

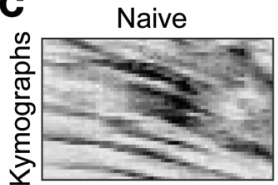

$\mathrm{CL}$

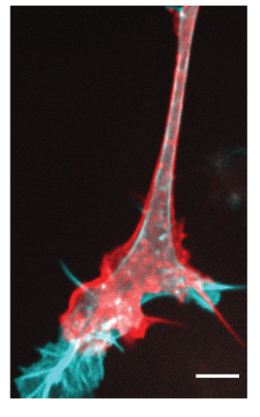

$\mathrm{CL}$

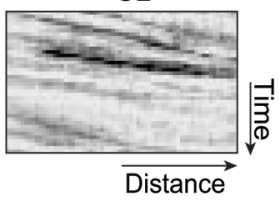

D

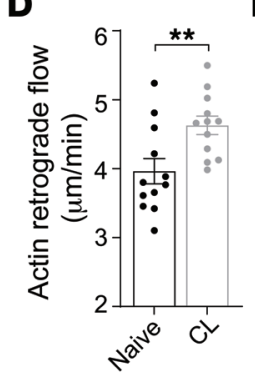

E

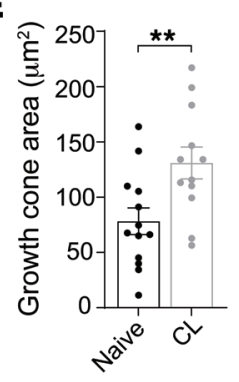

$\mathbf{F}$

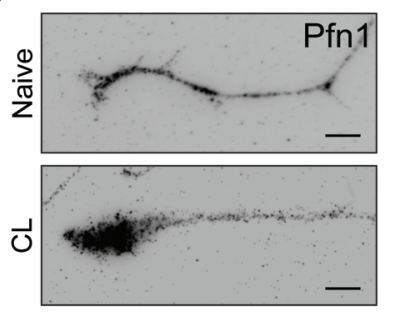

G

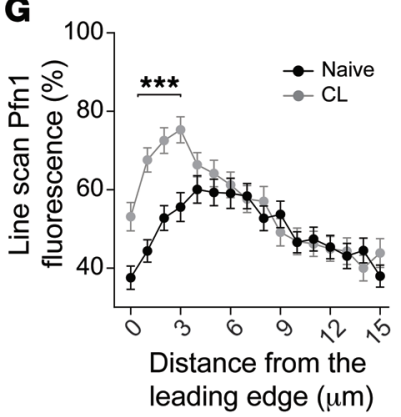

H

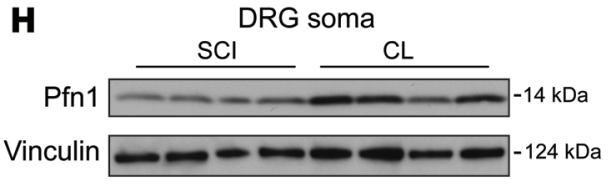

I

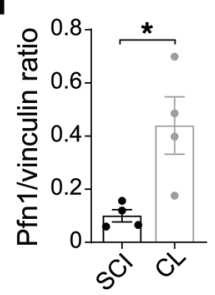

J

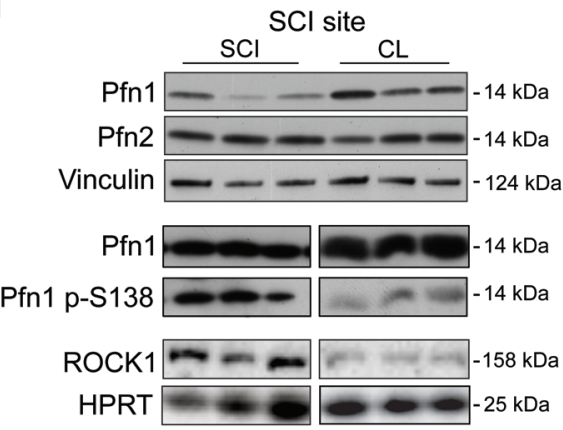

$\mathbf{K}$

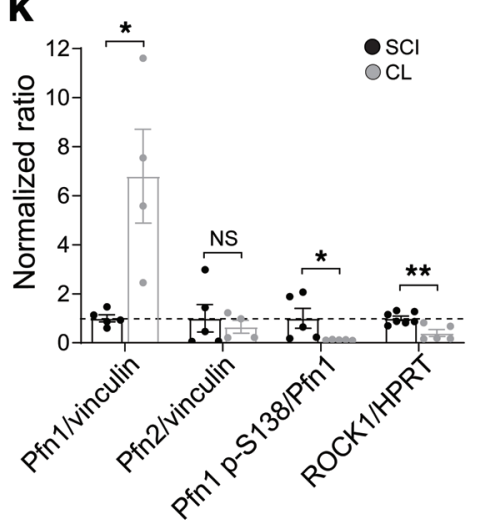

$\mathbf{L}$

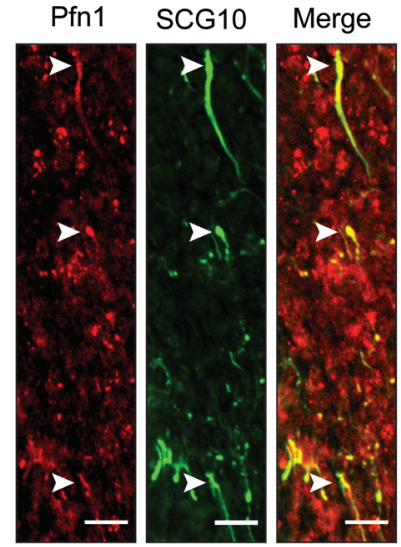

Figure 1. Active Pfn1 is increased after conditioning lesion (CL). (A) Representation of SCl and of the CL paradigm (left and right to dashed line, respectively). In CL, a sciatic nerve injury (1) is performed 1 week prior to $\mathrm{SCI}(2)$, potentiating regeneration of central DRG axons (right green line, rostral to SCI). Western blot analyses of the dorsal SCI site and of DRG (blue rectangles) were performed. (B) Live-cell imaging of LifeAct-GFP in growth cones of naive and conditioned adult DRG neurons. Scale bars: $4 \mu \mathrm{m}$. (C) Kymographs related to B. (D) Quantification of actin retrograde flow and (E) growth cone area related to B. Data represent mean \pm SEM ( $n=12-13$ growth cones/condition). ${ }^{* *} P<0.01$ by Student's $t$ test. (F) Pfn1 staining in growth cones of cultured naive and conditioned DRG neurons. Scale bars: $5 \mu \mathrm{m}$. (G) Quantification of line scans of Pfn1 fluorescence in relation to distance from growth cone leading edge related to F. Data represent mean \pm SEM ( $n=48-57$ neurons/condition). ${ }^{* *} P<0.001$ by 2-way ANOVA with Sidak's post hoc test. (H) Western blot and (I) respective quantification showing Pfn1 levels in DRG of rats with SCI or CL. Vinculin was used as control. Data represent mean \pm SEM $(n=4$ animals/condition). ${ }^{*} P<0.05$ by Student's $t$ test. (J) Western blot and (K) respective quantification showing Pfn1, Pfn1 p-S138, ROCK1, and Pfn2 levels in samples from the dorsal SCl site (horizontal blue rectangle in A), 1 week after SCI or CL. HPRT and vinculin were used as controls. Data represent mean \pm SEM ( $n=4-7$ animals/condition). ${ }^{*} P<0.05,{ }^{*} P<0.01$ by Student's $t$ test. NS, not significant. (L) Pfn1 immunofluorescence (red) in sensory SCG10-positive axons (green) in a CL spinal cord. Arrowheads highlight growth cones. Scale bars: $20 \mu \mathrm{m}$. 
A Naive

\begin{tabular}{rlll} 
TIMELINE & Day 0 & Day 1 & Day 2 \\
\cline { 2 - 3 } Procedure & $\begin{array}{l}\text { DRG neuron } \\
\text { nulture and } \\
\text { nucleofection }\end{array}$ & Plating & Fixation
\end{tabular}

B

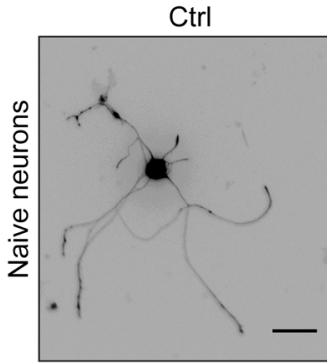

E
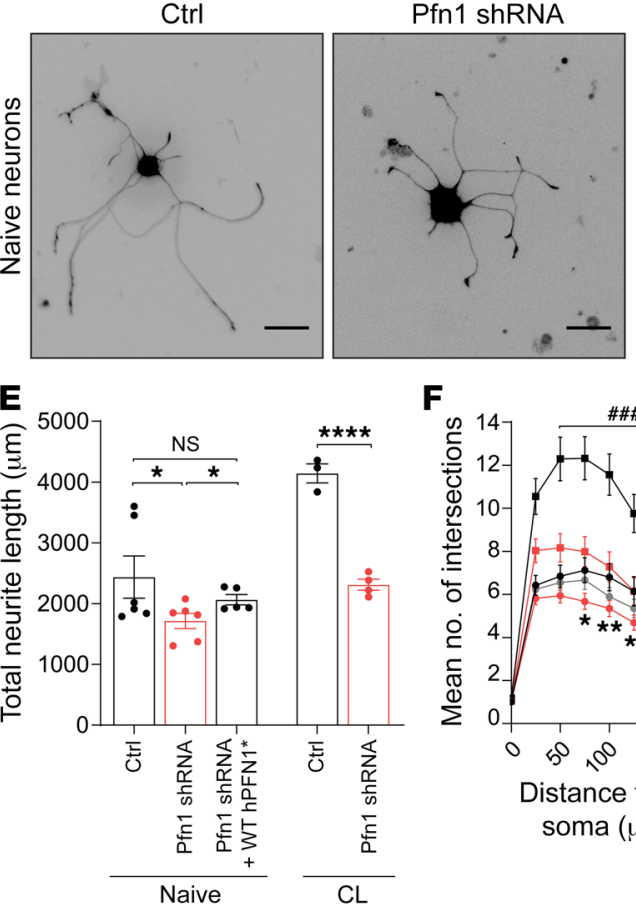

$\mathbf{F}$
C

$$
\begin{aligned}
& \begin{array}{clll}
\text { Timeline } & \text { Day }-7 & \text { Day } 0 & \text { Day } 1 \text { Day } 2 \\
\cline { 2 - 3 } & \text { Procedure } \\
\text { nerve } & \begin{array}{l}
\text { DRG neuron } \\
\text { culture and }
\end{array} & \text { Plating Fixation }
\end{array} \\
& \text { transection nucleofection }
\end{aligned}
$$

D

+ WT hPFn1*
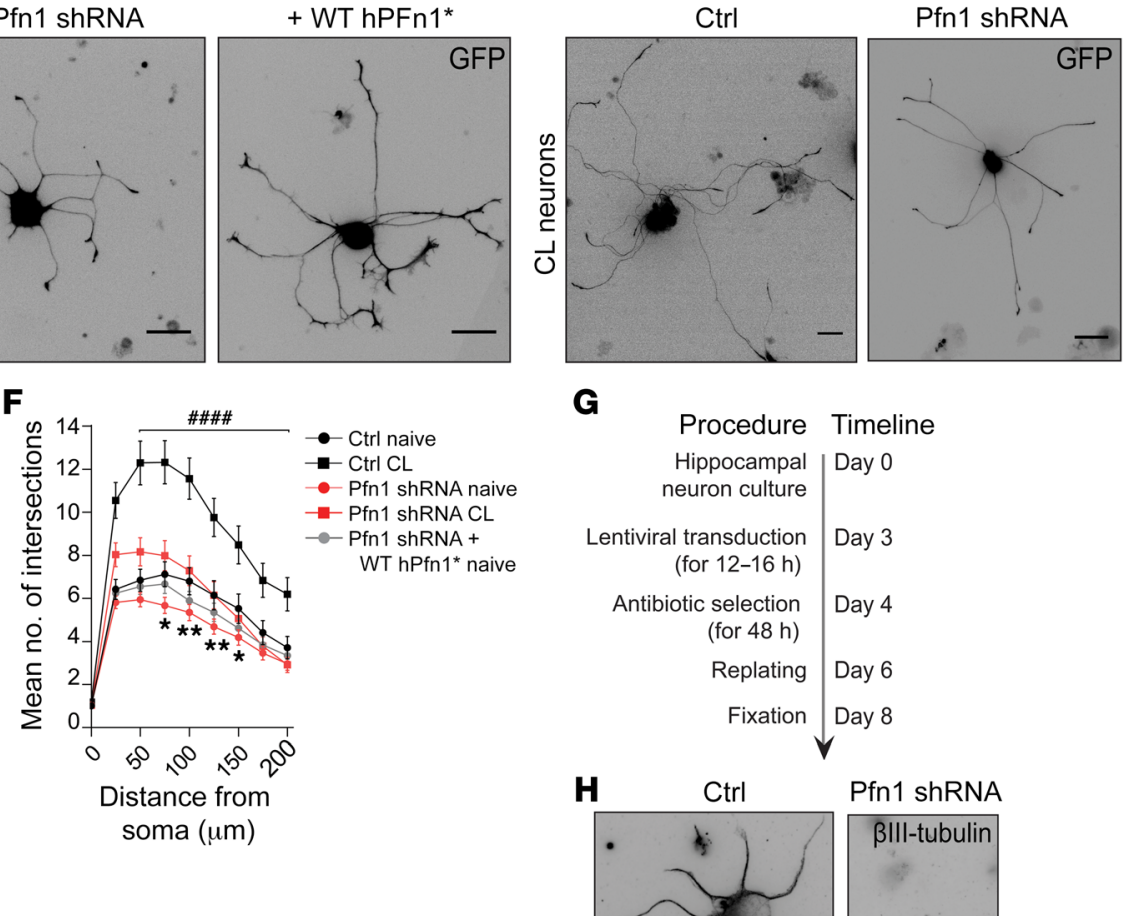

G

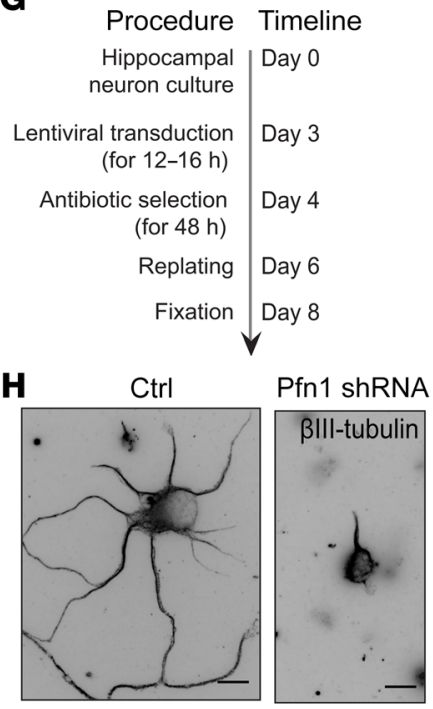

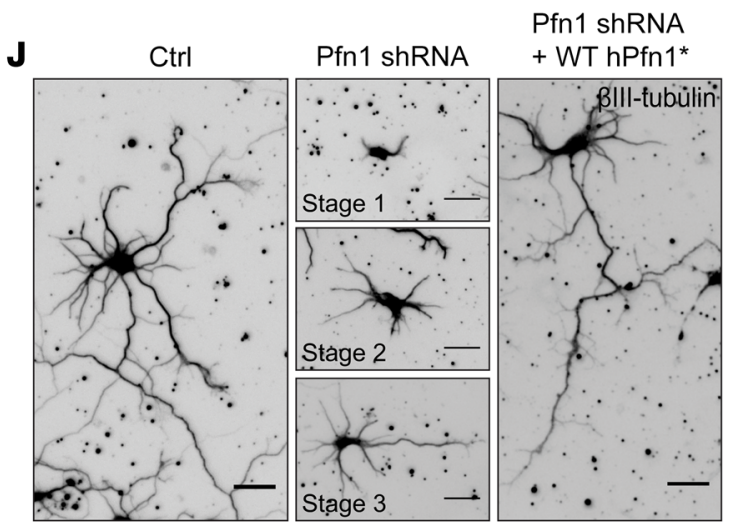
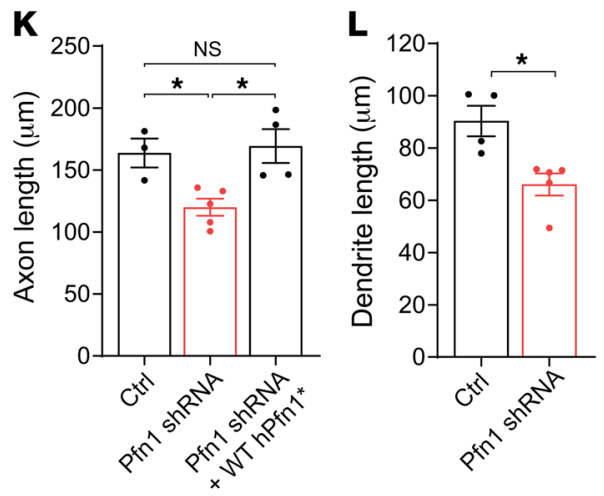

Figure 2. Pfn1 downregulation impairs axonal growth in vitro in different neuronal types and developmental stages. (A) Timeline of naive DRG neuron cultures. (B) GFP-expressing naive adult DRG neurons transfected with control empty (Ctrl) or Pfn1 shRNA plasmid. (C) Timeline of conditioned DRG neuron cultures. (D) GFP-expressing conditioned DRG neurons transfected with Ctrl or Pfn1 shRNA plasmid. Scale bars in B and D: $70 \mu \mathrm{m}$. (E) Total neurite length related to $\mathbf{B}$ and $\mathbf{D}$. Data represent mean \pm SEM ( $n=3-6$ independent samples/condition; 6-36 neurons/sample). ${ }^{*} P<0.05 ;{ }^{* * * *} P<0.0001 ;$ by Student's $t$ test. NS, not significant. (F) Branching analysis related to $\mathbf{E}$. Data represent mean $\pm \mathrm{SEM} .{ }^{*} P<0.05,{ }^{* *} P<0.01$ refers to Ctrl versus Pfn1 shRNA of naive DRG neurons; \#\#\# $P<0.0001$ refers to Ctrl versus Pfn1 shRNA of CL DRG neurons; 2-way ANOVA with Tukey's post hoc test. (G) Timeline for Pfn1 downregulation in DIV3 hippocampal neurons using lentiviral infection. (H) $\beta$ III-tubulin in hippocampal neurons after lentiviral expression of control empty (Ctrl) or Pfn1 shRNA plasmid. Scale bars: $10 \mu \mathrm{m}$. (I) Timeline for Pfn1 downregulation in DIVO hippocampal neurons. (J) BIII-tubulin in DIV4 hippocampal neurons expressing a control empty (Ctrl) or a Pfn1 shRNA plasmid. Middle panels (Pfn1 shRNA) show representative images of stage 1 to 3 hippocampal neurons. Scale bars: $30 \mu \mathrm{m}$ (Ctrl and Pfn1 shRNA + WT hPfn1*) and $20 \mu \mathrm{m}$ (Pfn1 shRNA). (K) Axonal length related to J. Data represent mean \pm SEM ( $n=3-5$ independent samples/condition; 11-26 neurons/sample). ${ }^{*} P<0.05$ by 1-way ANOVA Tukey's post hoc test. NS, not significant. (L) Dendritic length of DIV7 hippocampal neurons expressing control empty (Ctrl) or Pfn1 shRNA plasmid. Data represent mean \pm SEM ( $n=4-5$ independent samples/condition; 3-25 neurons/sample). ${ }^{*} P<0.05$ by Student's $t$ test. All rescue experiments were performed using shRNA-resistant WT Pfn1 (WT hPfn $1 *$ ). 
A
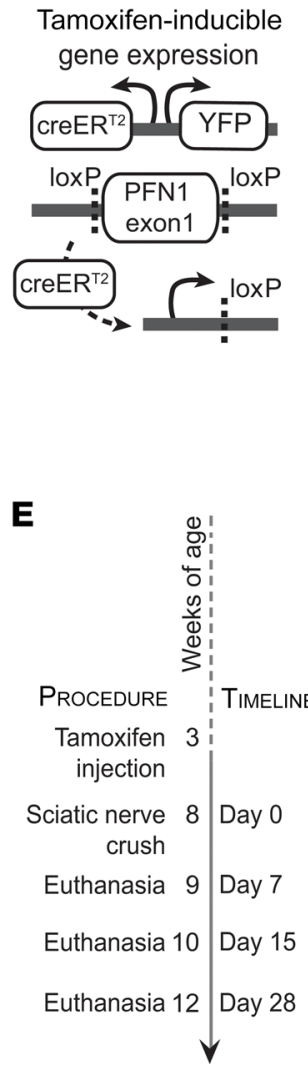
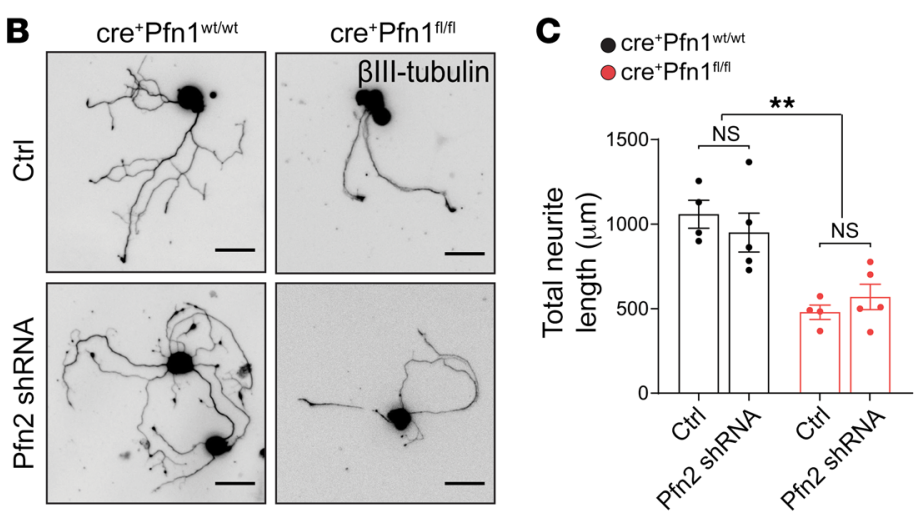

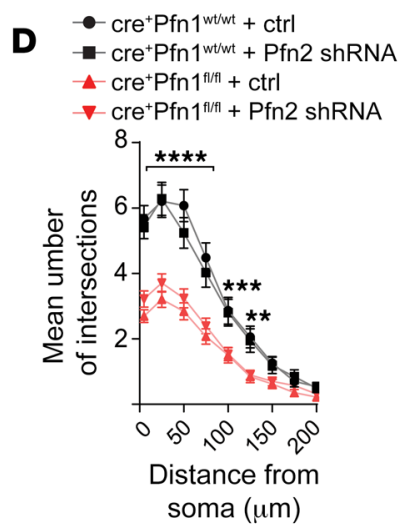

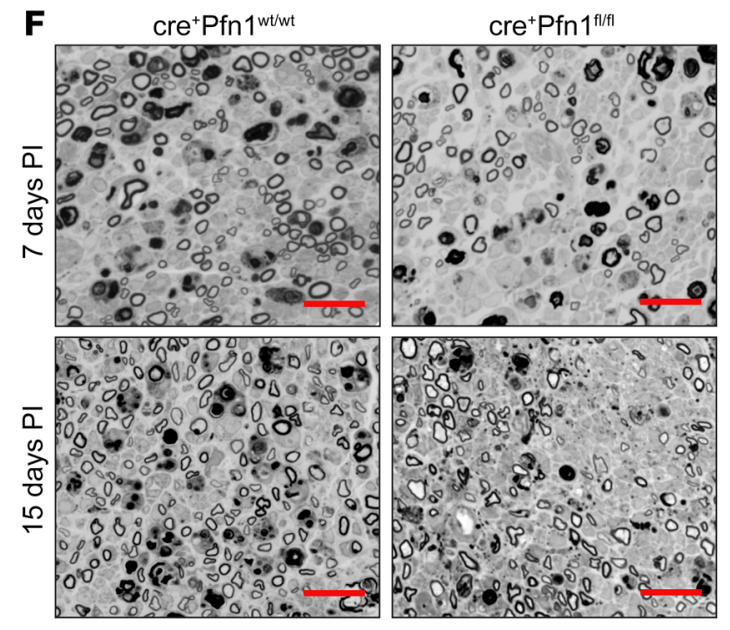

G

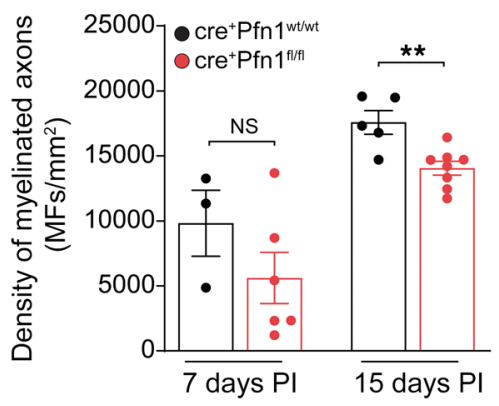

H

cre'Pfn1wtwt

$\mathrm{cre}^{+} \operatorname{Pfn} 1^{\text {tr/1 }}$
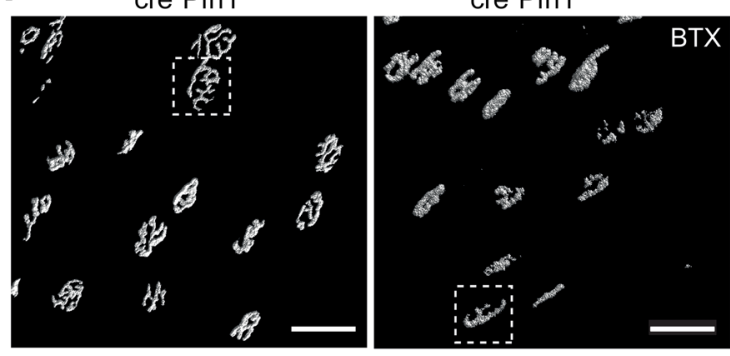

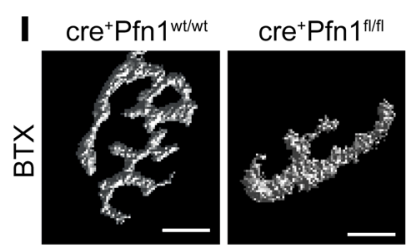

$3 \mathrm{D}$ rendered
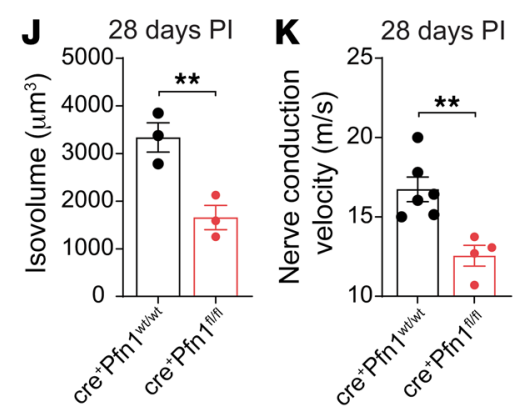

$\mathbf{L}$

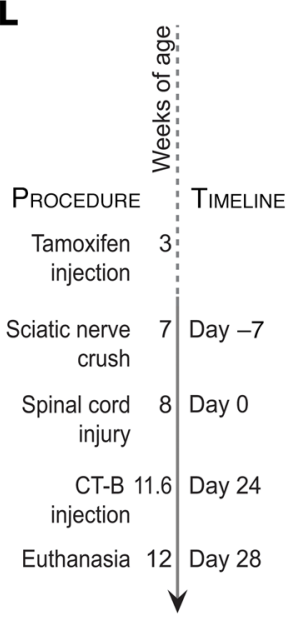

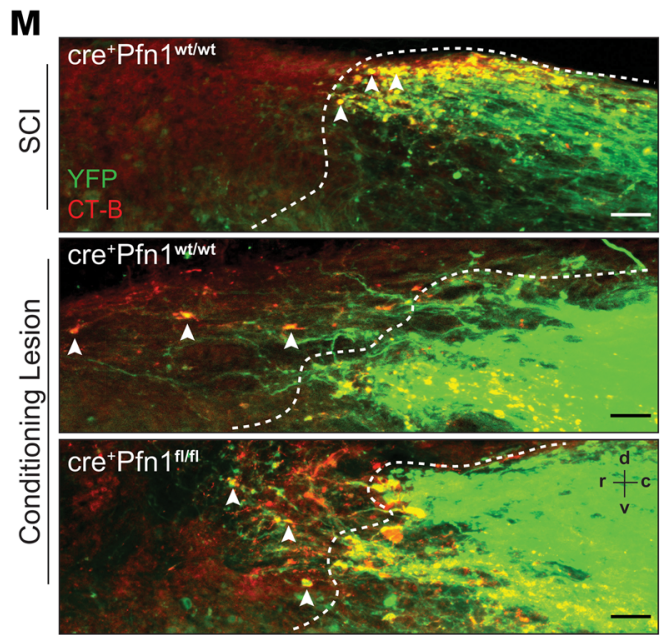

$\mathbf{N}$

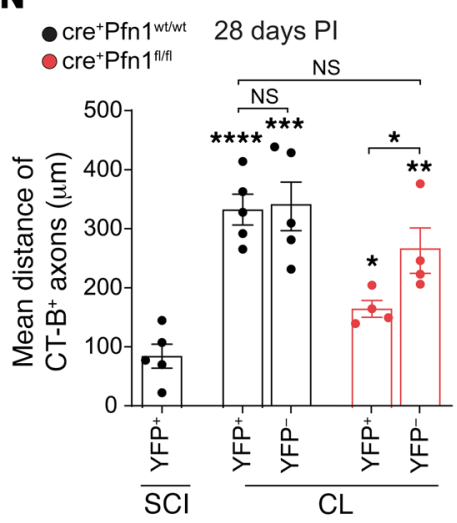


Figure 3. Pfn1 depletion in vivo decreases axonal regeneration and functional recovery. (A) Neuronal Thy1 promoter drives Cre recombinase and YFP expression in cre $+P f n 1$ mice after tamoxifen administration, leading to $P f n 1$ exon1 excision. (B) $\beta / l l$-tubulin staining of cre+Pfn1 adult DRG neurons in the presence or absence of a Pfn2 shRNA-expressing plasmid. Scale bars: $50 \mu \mathrm{m}$. (C) Total neurite length and (D) branching analysis related to B. Only YFP+ (Pfn1-KO) neurons were quantified. Data represent mean \pm SEM ( $n=4-5$ independent samples/condition; 5-35 neurons/sample). ${ }^{* *} P$ $<0.01$; ${ }^{* *} P<0.001 ;{ }^{* * *} P<0.0001$ by 1 -way ANOVA with Tukey's post hoc test (C) or 2-way ANOVA with Tukey's post hoc test (D). (E) Strategy to assess PNS regeneration. (F) PPD-stained sciatic nerves from cre ${ }^{+} \mathrm{Pfn} 1$ mice, 7 and 15 days postinjury (PI). Scale bars: $20 \mu \mathrm{m}$. (C) Myelinated axon density related to $\mathbf{F}$. Data represent mean \pm SEM ( $n=3-8$ animals/ condition). ${ }^{* *} P<0.01$ by Student's $t$ test. MFs, myelinated fibers. (H) $3 D$ surface-rendered reconstructions of NMJs fluorescently labeled with $\alpha$-bungarotoxin (BTX). Scale bars: $50 \mu \mathrm{m}$. (I) Zoom-ins of $\mathbf{H}$. Scale bars: 10 $\mu \mathrm{m}$. (J) Volume quantification of NMJs (28 days PI). Data represent mean \pm SEM ( $n=3$ animals/condition). ${ }^{*} P<0.01$ by Student's $t$ test. (K) Motor nerve conduction velocity ( 28 days $\mathrm{PI})$. Data represent mean $\pm \operatorname{SEM}(n=$ 4-6 animals/condition). ${ }^{* *} P<0.01$ by Student's $t$ test. (L) Strategy to assess CNS regeneration. (M) YFP ${ }^{+}$(green)/CT-B+ (red) axons (arrowheads) in spinal cord following $\mathrm{SCl}$ in $\mathrm{Cre}^{+} \mathrm{Pfn} 1^{\text {wt/wt }}$ and $\mathrm{CL}$ in either $\mathrm{cre}^{+} \mathrm{Pfn} 1^{\mathrm{wt} / \mathrm{wt}}$ or $\mathrm{cre}^{+} P f n 1^{f l / f l}$ mice. Scale bars: $50 \mu \mathrm{m}$. Dashed line, lesion border; r, rostral; c, caudal; d, dorsal; v, ventral. (N) Quantification of mean growth distance of $\mathrm{YFP}^{+}(\mathrm{Pfn} 1-\mathrm{KO})$ and $\mathrm{YFP}^{-}$ascending sensory axons (CT- $\mathrm{B}^{+}$axons) from the rostral end of the injured dorsal column tract. Data represent mean \pm SEM ( $n=4-5$ animals/condition). ${ }^{*} P<0.05$; ${ }^{* *} P<0.01 ;{ }^{* * *} P<0.001 ;{ }^{* * *} P<$ 0.0001 by 1 -way ANOVA with Tukey's post hoc test. NS, not significant.

we investigated if Pfn2 would also regulate neurite outgrowth in DRG neurons. Interestingly, downregulation of Pfn2 levels (Supplemental Figure 1, E and F) decreased neuronal growth cone area, although to a lower extent than that of Pfn1 (Supplemental Figure 1, G and H). However, Pfn2 downregulation did not change the elongation competence or branching of either $\mathrm{cre}^{+} \mathrm{Pfn} 1^{\mathrm{wt} / \mathrm{wt}}$ or $\mathrm{cre}^{+} \mathrm{Pfn} 1^{\mathrm{t} / \mathrm{fl}}$ naive DRG neurons (Figure 3, B-D). These observations suggest that brain-specific Pfn2 might play alternative functions in DRG neurons, unrelated to neurite growth.

Next, we determined whether the lack of Pfn1 is sufficient to impair axonal regeneration in vivo in 2 well-described paradigms leading to robust axonal regeneration: the sciatic nerve injury model and the CL model. Following sciatic nerve injury, axons successfully regenerate and remyelination occurs soon after injury. Upon crushing the sciatic nerve we counted myelinated axons distally to the lesion site at different time points (Figure 3E). At 7 days after injury, the density of myelinated fibers in $\mathrm{cre}^{+} \mathrm{Pfn} 1^{\mathrm{f} / \mathrm{fl}}$ mice showed an over $40 \%$ decrease in comparison with $\mathrm{cre}^{+} \mathrm{Pfn} 1^{\mathrm{wt} / \mathrm{wt}}$, mice, and at 15 days after injury the absence of Pfn1 led to a significant decrease in the number of myelinated axons (Figure 3, F and G). These results indicate an impaired axonal regenerative capacity in the absence of Pfn1 in vivo. Of note, no differences in remyelination were detected in $\mathrm{cre}^{+} \mathrm{Pfn} 1^{\mathrm{f} / \mathrm{fl}}$ mice, as assessed by g-ratio measurements at 15 and 28 days after injury (data not shown). At 28 days after injury, functional synaptic contacts - neuromuscular junctions (NMJs) - in the gastrocnemius muscle were evaluated through the analysis of acetylcholine receptor (AChR) clusters, using the postsynaptic marker $\alpha$-bungarotoxin. Analysis of $3 \mathrm{D}$ surface-reconstructed AChR clusters revealed that the structural volume and complexity were largely reduced in $\mathrm{cre}^{+} \mathrm{Pfn} 1^{\mathrm{fl} / \mathrm{fl}}$ mice (Figure $3, \mathrm{H}-\mathrm{J}$ ), pointing toward a delayed $\mathrm{NMJ}$ maturation in the absence of Pfn1. At the functional level, motor nerve conduction velocity showed a clear deficit in $\mathrm{cre}^{+} \mathrm{Pfn} 1^{\mathrm{f} / \mathrm{fl}}$ mice compared with cre $^{+} \mathrm{Pfn} 1^{\mathrm{wt} / \mathrm{wt}}$ mice (Figure $3 \mathrm{~K}$ ), supporting a decreased number of functional myelinated axons. Together, these results support the notion that the lack of Pfn1 results in defective axonal regeneration and functional recovery of damaged peripheral axons.

We also used the CL paradigm as an alternative in vivo model. In this experimental model, the enhanced regenerative capacity of the ascending dorsal column tract was assessed in mice, in which a sciatic nerve transection preceded an acute spinal cord lesion (dorsal hemisection) (Figure 3L). Cholera toxin B (CT-B) subunit, a tracer previously injected in the sciatic nerve, was used to visualize regenerating dorsal column ascending sensory axons (Figure $3 \mathrm{M}$ ). The injured dorsal column tract was clearly identified by the accumulation of YFP-expressing axons in the dorsal region of the thoracic spinal cord (Figure $3 \mathrm{M}$ ). Whereas dorsal column tract axons (yellow, highlighted with white arrowheads) accumulated in the lesion border of $\mathrm{cre}^{+} \mathrm{Pfn} 1^{\mathrm{wt} / \mathrm{wt}}$ mice with SCI (Figure 3M), long-distance regeneration (4.4-fold increase) was observed in $\mathrm{cre}^{+} \mathrm{Pfn} 1^{\mathrm{wt} / \mathrm{wt}}$ mice with CL (Figure $3 \mathrm{M})$. In sharp contrast, $\mathrm{cre}^{+} \mathrm{Pfn} 1^{\mathrm{fl} / \mathrm{fl}}$ mice with CL showed over $50 \%$ reduction in the mean regenerating distance (Figure 3M), with most axons already aborting their regeneration close to the injury border (Figure 3, M and N). As an internal control, CT- $\mathrm{B}^{+} \mathrm{YFP}^{-}$axons were measured, further supporting a Pfn1-specific effect (Figure $3 \mathrm{~N}$ ) in regulating axonal regeneration. These observations support the idea that Pfn1 is an important player for optimal axonal extension after injury in vivo.

$P f n 1$ regulates actin and MT dynamics in the growth cone, increasing axonal growth in vitro. Because the force required to power axonal growth and regeneration is regulated by cytoskeletal components at the distal tip of a growing neurite, we compared cytoskeletal dynamics in growth cones of adult DRG neurons from $\mathrm{cre}^{+} \mathrm{Pfn} 1^{\mathrm{wt} / \mathrm{wt}}$ and $\mathrm{cre}^{+} \mathrm{Pfn} 1^{\mathrm{f} / \mathrm{fl}}$ mice. Phalloidin staining revealed that $\mathrm{cre}^{+} \mathrm{Pfn} 1^{\mathrm{f} / \mathrm{fl}}$ sensory neurons extended smaller growth cones (Figure 4, A and B), in support of our data using shRNA-mediated downregulation of Pfn1 in hippocampal neurons (Supplemental Figure 1, G and $\mathrm{H}$ ). Although we observed a similar number of filopodia in $\mathrm{cre}^{+} \mathrm{Pfn} 1^{\mathrm{f} / \mathrm{fl}}$ and $\mathrm{cre}^{+} \mathrm{Pfn} 1^{\mathrm{wt} / \mathrm{wt}}$ animals (Figure 4, A and $\mathrm{C}$ ), the existing filopodia in $\mathrm{cre}^{+} \mathrm{Pfn} 1^{\mathrm{fl} / \mathrm{ll}}$ growth cones were significantly shorter compared with controls (Figure 4, A and D). Furthermore, actin dynamics assessed by measuring the velocity of actin retrograde flow was reduced by $30 \%$ in growth cones of adult $\mathrm{cre}^{+} \mathrm{Pfn} 1^{\mathrm{f} / \mathrm{l} / \mathrm{l}}$ DRG neurons (Figure 4, E-G) and was reverted upon reexpression of WT hPfn1 (Figure 4, E-G). Interestingly, in addition to actin dynamics, MT growth speed, measured in growth cone filopodia, was also affected by Pfn1 deletion. In $\mathrm{cre}^{+} \mathrm{Pfn} 1^{\mathrm{fl} / \mathrm{fl}}$ DRG neurons, the end-binding protein 3 (EB3) comet speed was $50 \%$ diminished (Figure $4, \mathrm{H}^{-} \mathrm{J}$ ), with growing MTs presenting a decreased growth length (Supplemental Figure 2A), without a significant difference in the duration of growth (Supplemental Figure 2B). The defect in MT dynamics in $\mathrm{cre}^{+} \mathrm{Pfn} 1^{\mathrm{f} / \mathrm{fl}} \mathrm{DRG}$ neurons was completely reversed by the expression of WT hPfn1 (Figure 4, $\mathrm{H}-\mathrm{J}$ ). Of note, when similar analyses were performed in the axonal shaft, Pfn1 deletion did not significantly affect EB3 comet speed (Figure 4J), supporting a possible compartment-specific function of Pfn1 in the growth cone. Combined, these data suggest that in the absence of Pfn1, MTs polymerize at lower rates, likely leading 
A

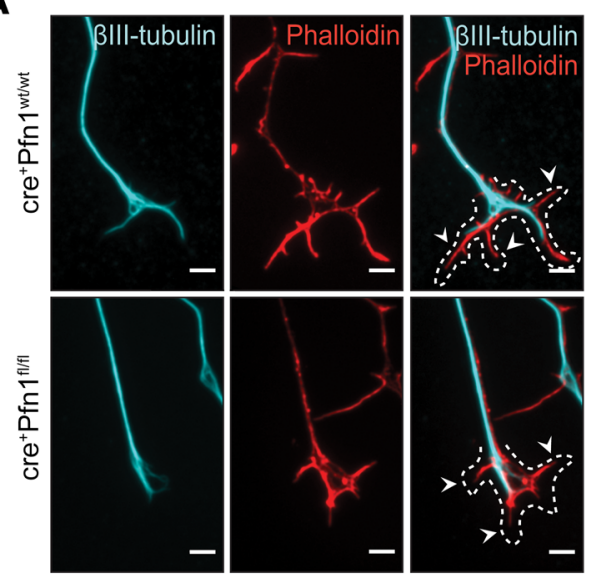

B

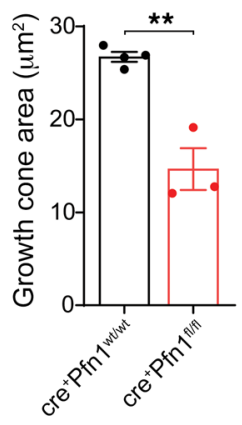

C

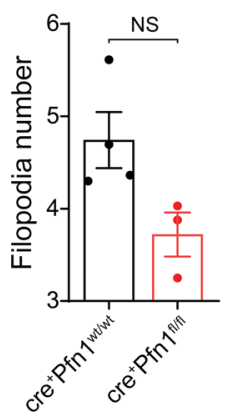

D

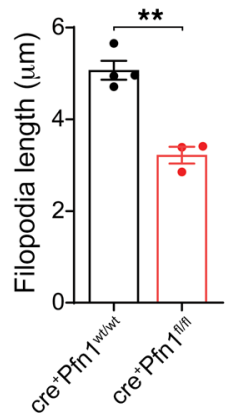

E
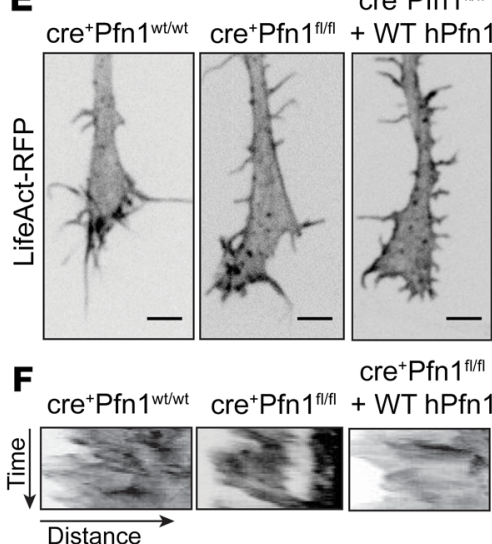
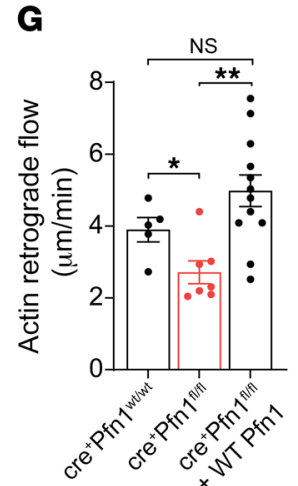

H
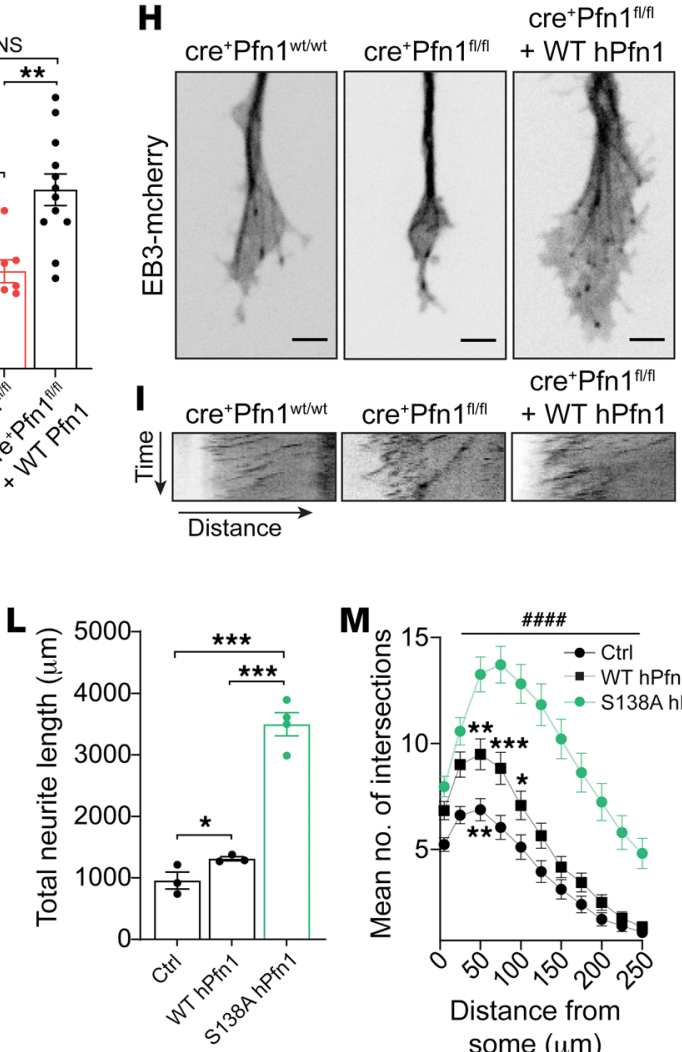

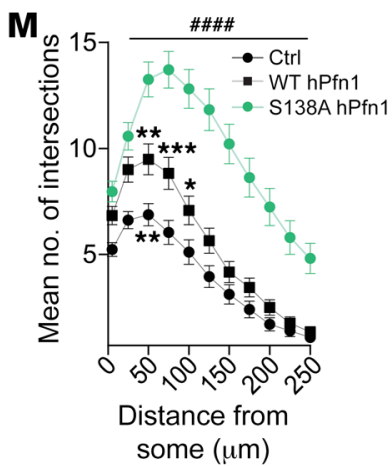

N $20 \mu \mathrm{g} / \mathrm{mL}$ aggrecan

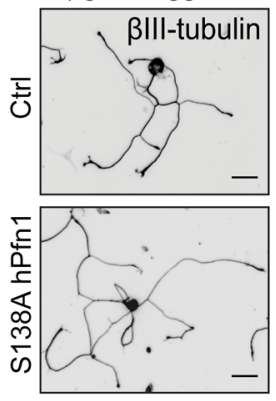

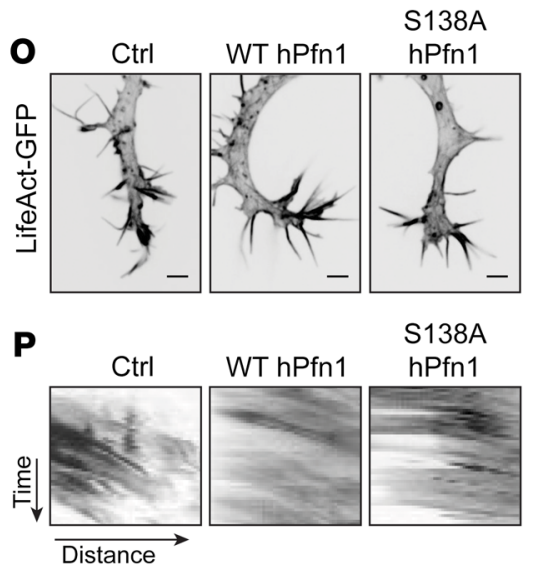
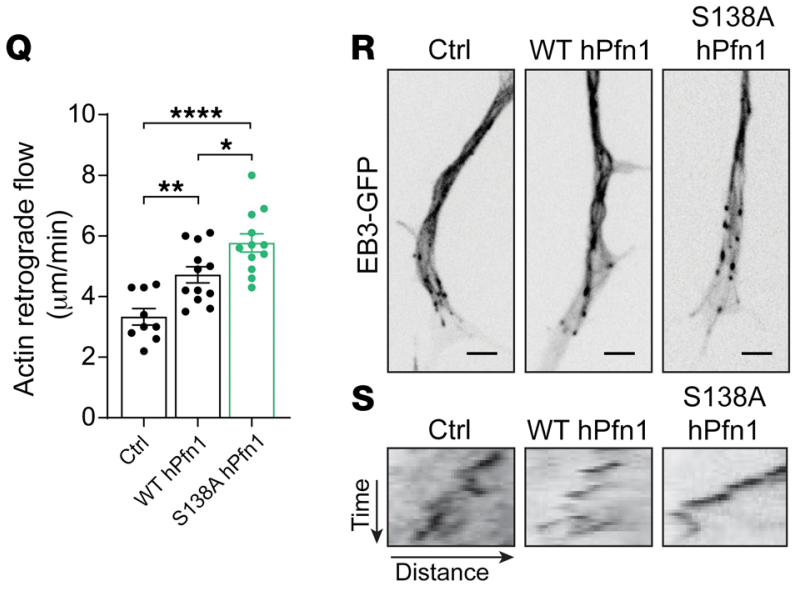

$\mathbf{T}$

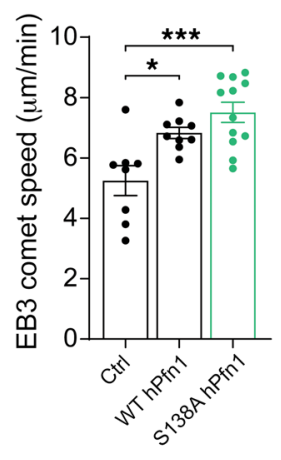


Figure 4. Pfn1 regulates actin and MT dynamics in growth cones. (A) BIII-tubulin (cyan) and actin (red) in cre+Pfn1 DRG growth cones. Scale bars: $3 \mu \mathrm{m}$. Dashed line, cone area; arrowheads, filopodia. (B) Growth cone area, (C) filopodia number, and (D) length related to $\mathbf{A}$. Data represent mean \pm SEM ( $n=32-40$ neurons/animal, 3-4 animals/condition). ${ }^{*} P<0.01$ by Student's $t$ test. (E) LifeAct-RFP in cre+Pfn1 DRG growth cones. Scale bars: $3 \mu \mathrm{m}$. (F) Kymographs and (G) actin flow quantification related to $\mathbf{E}$. Data represent mean \pm SEM ( $n=5-12$ filopodia/condition; representative of 3 to 4 growth cones/condition. ${ }^{*} P<0.05 ;{ }^{*} P<0.01$ by 1 -way ANOVA with Tukey's post hoc test. (H) EB3-mCherry in cre+Pfn1 DRG growth cones. (I) Kymographs and (J) EB3 speed quantification related to $\mathbf{H}$ in growth cones and shaft. Data represent mean \pm SEM ( $n=3-7$ growth cones/condition). ${ }^{* *} P<0.01$ by 1 -way ANOVA with Tukey's post hoc test. (K) $\beta$ III-tubulin in WT and S138A hPfn1 DRG neurons. Scale bar: $80 \mu \mathrm{m}$. (L) Total neurite length and $(\mathbf{M})$ branching related to $\mathbf{K}$. For $\mathbf{L}$ and $\mathbf{M}$, data represent mean \pm SEM. (L) ${ }^{*} P<0.05$ and ${ }^{* *} P<0.001, n=3-4$ independent samples/condition; $13-31$ neurons/sample. (M) ${ }^{*} P<0.05,{ }^{* *} P<0.01,{ }^{* *} P<0.001$ refers to $C$ trl versus WT hPfn1; \#\#\#\#<0.0001 refers to Ctrl versus S138A hPfn1; 2-way ANOVA with Tukey's post hoc test. $n=100-113$ neurons/condition. (N) BIII-tubulin in S138A hPfn1 DRG neurons cultured on aggrecan. Scale bars: $50 \mu \mathrm{m}$. (O) LifeAct-GFP, (P) kymographs, and (Q) actin flow quantification in growth cones related to $\mathbf{K}$. (R) EB3-GFP, (S) kymographs, and (T) EB3 speed quantification in growth cones related to $\mathbf{K}$. Scale bars ( $\mathbf{O}$ and $\mathbf{R}): 3 \mu \mathrm{m}$. (Q and $\mathbf{T})$ Data represent mean \pm SEM ( $n=8-12$ growth cones/ condition). ${ }^{*} P<0.05$, ${ }^{* *} P<0.01,{ }^{* *} P<0.001,{ }^{* * *} P<0.0001$ by 1 -way ANOVA with Tukey's post hoc test. NS, not significant.

to shorter MTs. Similar results were obtained in growth cones of embryonic hippocampal neurons, which also showed over $25 \%$ decreased actin retrograde flow upon shRNA-mediated knockdown of Pfn1 (Supplemental Figure 2, C-E), and significantly reduced growth speed and length of polymerizing MTs onto the peripheral membrane edge (Supplemental Figure 2, F-I). Of note, in these live-cell experiments, fluorescently tagged LifeAct and EB3 were imaged in growth cones of stage 3 hippocampal neurons. However, a considerable number of stage 1 neurons was consistently observed in the Pfn1-shRNA condition (Supplemental Figure 2, C and F, middle). Thus, acute Pfn1 depletion induced defective actin and MT dynamics, which in many neurons led to an arrest of axonal outgrowth.

The CL model suggests that an increased activity of Pfn1 is necessary to achieve a high regenerative capacity (Figure 1). Therefore, we hypothesized that the delivery of active Pfn1 might persistently accelerate cytoskeletal dynamics in the growth cone, ultimately leading to axonal elongation. To test this hypothesis, we generated a constitutively active nonphosphorylatable Pfn1 mutant (S138A hPfn1). In adult sensory DRG neurons, overexpression of S138A hPfn1 elicited a 2.6-fold increase in total neurite length (Figure 4, $\mathrm{K}$ and $\mathrm{L}$ ) and a substantial rise in the mean number of branches (Figure $4 \mathrm{M}$ ), whereas only a small increase in both parameters was observed upon overexpression of WT hPfn1. Of note, overexpression efficiency was similar for both WT hPfn1 and S138A hPfn1 (Supplemental Figure 2, J and K). Importantly, S138A hPfn1 was also effective in promoting growth (1.4-fold increase) of adult DRG neurons cultured on inhibitory substrates such as aggrecan (Figure $4 \mathrm{~N}$ ). These data demonstrate that active $\mathrm{Pfn} 1$ is an important enhancer of axonal growth under permissive as well as inhibitory conditions. Because Pfn1-depleted neurons show abnormal cytoskeletal dynamics in their growth cones, we hypothesized that increased Pfn1 activity may promote not only actin but also MT dynamics. In support of our hypothesis, overexpression of both WT and S138A hPfn1 significantly increased actin retrograde flow (Figure 4, O-Q) and EB3 comet speed (Figure 4, $\mathrm{R}-\mathrm{T}$ ) at the growing tip, with S138A hPfn1 presenting a significantly higher effect. Importantly, similar results were obtained in hippocampal neuron cultures, suggesting that S138A hPfn1 affects these parameters in different neuronal populations. Specifically, S138A hPfn1-expressing DIV4 hippocampal neurons presented longer axons (Supplemental Figure 2, L and M) and increased actin (Supplemental Figure 2N) and MT dynamics (Supplemental Figure 2, $\mathrm{O}$ and $\mathrm{P}$ ). Together, our data suggest that $\mathrm{S} 138 \mathrm{~A} \mathrm{hPfn} 1$ is a robust activator of actin and MT dynamics in the growth cone, and of axonal growth capacity.

Regulation of the MT cytoskeleton in growth cones and promotion of axonal elongation by S138A hPfn1 are facilitated by direct MT binding and interaction with formins. Pfn1 has a plethora of ligands, including actin, poly-proline-containing proteins, and $\mathrm{PIP}_{2}$ at the plasma membrane (ref. 28 and Figure 5A). In addition to the above ligands, Pfn1 is able to bind directly to MTs in vitro (25), through residues found mutated in ALS patients, including G118 (Figure 5A and ref. 33). Given this evidence, we explored the possibility that in neurons, S138A hPfn1 might increase MT growth speed through direct binding to MTs. For this purpose, we expressed in hippocampal neurons the MT binding-deficient G118V hPfn1 (25, 33) in a constitutively active S138A hPfn1 backbone (G118V/S138A hPfn1). Of note, the speed of actin retrograde flow in growth cones is still powered by the expression of G118V hPfn1 (Supplemental Figure 2, Q-S), allowing the uncoupling of the effect of this mutant on MT growth from a possible effect on actin dynamics. Interestingly, expression of the double mutant, G118V/S138A hPfn1, increased MT growth speed to a comparable extent as the single mutant S138A hPfn1 (Figure 5, B-D). Thus, S138A hPfn1 increases MT growth through a mechanism that does not involve direct MT binding. To understand if Pfn1 molecular partners participate in the effect of constitutively active Pfn1 on MT dynamics, we explored the relevance of the Pfn1 poly-proline binding domain. Overexpression of the double mutant H134S/S138A hPfn1, i.e., poly-proline-binding-deficient hPfn1, decreased S138A hPfn1's ability to promote MT growth speed (Figure 5, B-D). These data support the notion that in growth cones, S138A hPfn1 increases MT dynamics through a poly-proline-containing partner. Pfn1 works closely with formins that bear a proline-rich formin homology domain 1 (FH1) and function as actin assembly factors assisting the formation of unbranched actin filaments (34). Whereas FH1 speeds up actin assembly by recruiting Pfn-bound actin monomers to the vicinity of the barbed end through its proline-rich motifs, formin homology domain 2 (FH2), after nucleating actin dimers, remains attached to the actin filament to assist its elongation (35). In addition to regulating actin filament growth, formins also bind MTs and influence their stability independently of actin binding $(36,37)$. We analyzed if formins might mediate the effect of S138A hPfn1 in promoting MT growth speed in neuronal growth cones. Inhibition of formins with a small-molecule inhibitor of the FH2 domain (SMIFH2) (38) was sufficient to prevent constitutively active S138A hPfn1 from powering MT growth speed (Figure 5, B-D). Thus, our data show that S138A hPfn1 increases MT dynamics through a formin-dependent mechanism. 
A

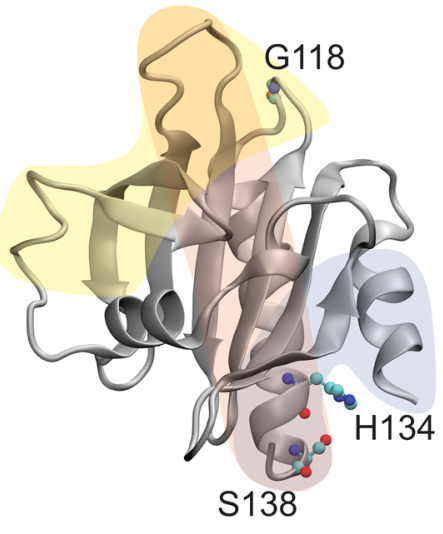

B

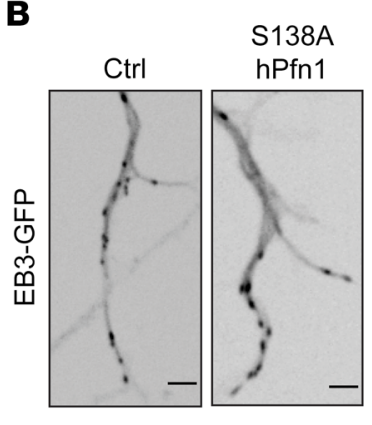

c
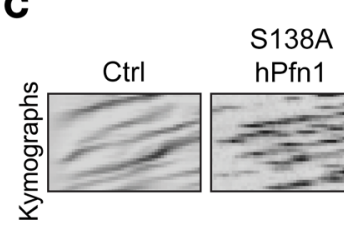

hPfn1

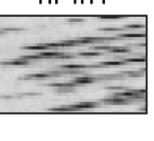

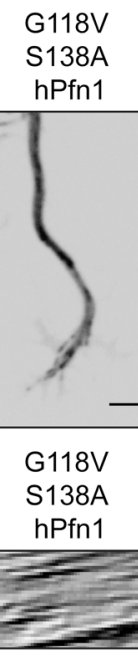
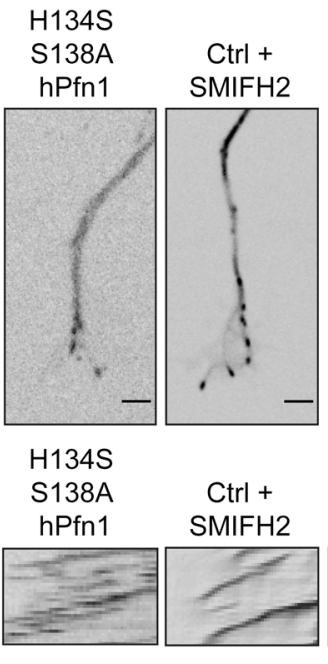

S138A

hPfn $1+$ SMIFH2

D
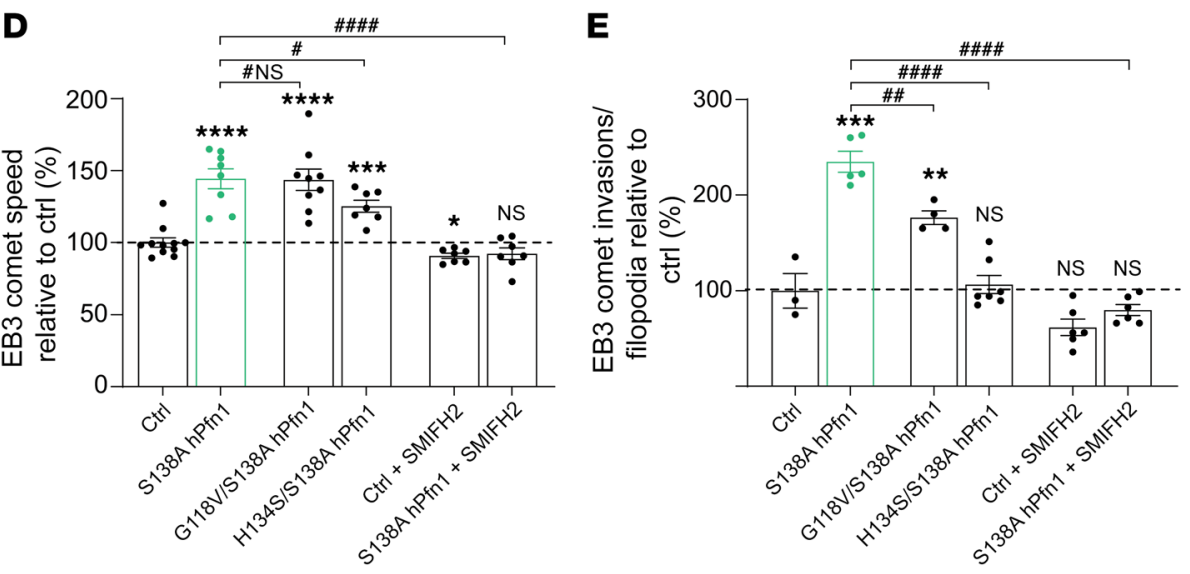

$\mathbf{F}$

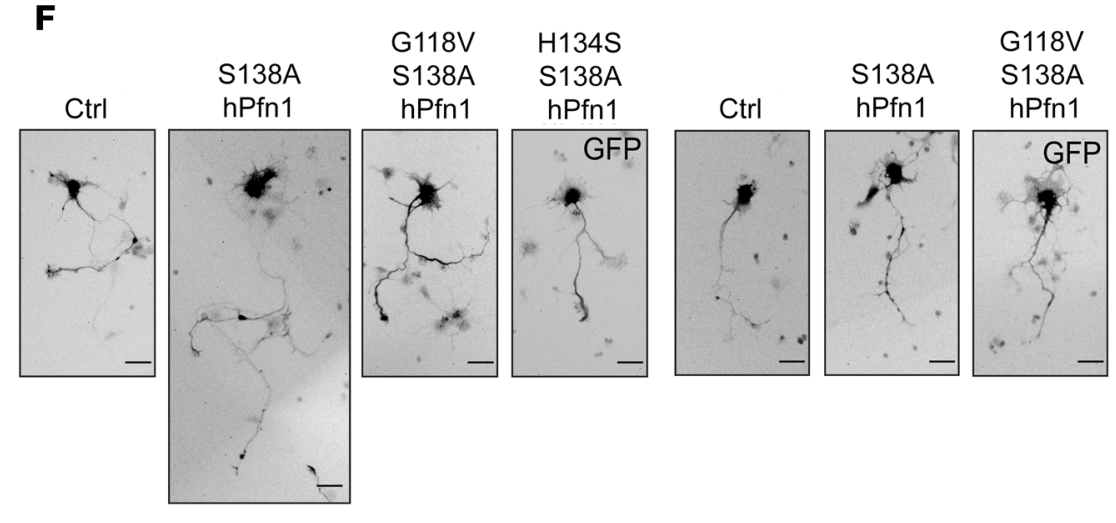

G

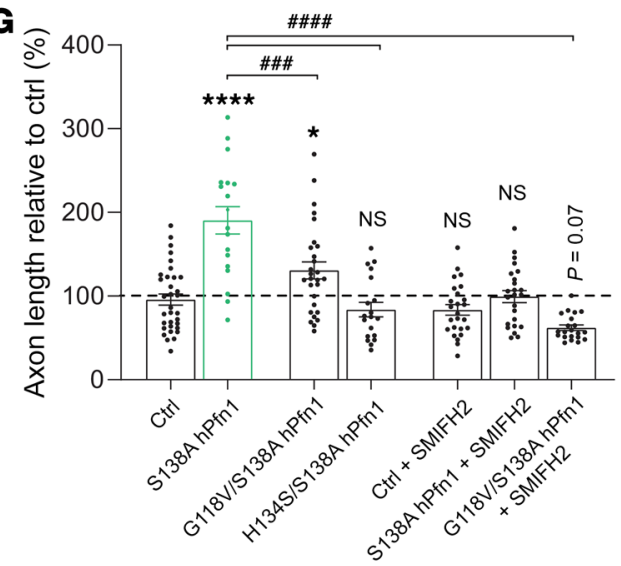

Figure 5. S138A Pfn1 enhances MT dynamics via direct MT binding and formins. (A) Crystal structure of hPfn1 (PDB code: 1cf0). Residues G118 (MT-binding), H134 (poly-proline-binding), and S138 (ROCK phosphorylation site, mediating inactivation of Pfn1-related functions) are highlighted. Actin-, poly-proline-, and $\mathrm{PI}(4,5) \mathrm{P}_{2}$-binding regions of $\mathrm{Pfn} 1$ are shadowed in light yellow, gray, and red, respectively (adapted from ref. 66). (B) Live-cell imaging of EB3-GFP in hippocampal neurons transfected with EB3-GFP and either a control empty vector (Ctrl) or plasmids expressing S138A hPfn1 or S138A Pfn1 mutants (G118V/S138A or H134S/S138A hPfn1); Ctrl and S138A hPfn1 treated with SMIFH2 are also shown. Scale bars: $2 \mu \mathrm{m}$. (C) Kymographs related to B. (D) Analysis of MT growth speed and (E) EB3 comet invasion frequency per filopodia. In D and E, data represent mean \pm SEM ( $n=7-11$ [D] and $n=3-7$ [E]

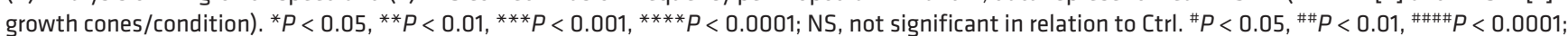
\#NS, not significant in relation to S138A hPfn1. (F) GFP+ hippocampal neurons transfected with either a control empty vector (Ctrl) or plasmids expressing different hPfn1 mutants, either untreated or treated with SMIFH2, whenever indicated. Scale bars: $30 \mu \mathrm{m}$. (G) Quantification of axonal length related to F Data represent mean \pm SEM ( $n=18-33$ neurons/condition; representative of 3-5 independent experiments/condition). ${ }^{*} P<0.05,{ }^{* * * *} P<0.0001$; NS, not

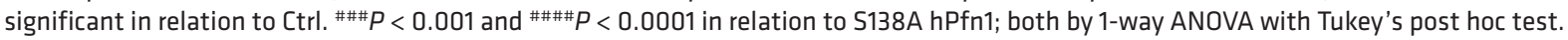


In the central domain of the growth cone, axonal MT bundles terminate and few explorative MTs enter within filopodia of the growth cone peripheral domain. These MTs can lead the advance of the shaft MT bundles, culminating in axonal growth (7, 39-41). Given this evidence, to further dissect the effect of Pfn1 in the growth cone, we assessed filopodia invasion by MTs. Constitutively active S138A hPfn1 strongly enhanced the localization of growing MTs to growth cone filopodia (Figure $5 \mathrm{E}$ ). This effect was sharply diminished when direct Pfn1 binding to MTs was abolished through the use of the double mutant G118V/S138A hPfn1 and was totally reverted by a poly-proline-binding-deficient mutant (H134S/S138A hPfn1) or inhibition of formins (Figure 5E). Taken together, our data show that S138A hPfn1 increases MT growth speed through a formin-dependent mechanism, whereas localization of growing MTs to filopodia is promoted both through direct MT binding and interaction with formins. In line with the above findings, the ability of constitutively active Pfn1 to promote axonal growth was severely impaired by a mutation either in the MT-binding region (G118V/S138A hPfn1) or in the poly-proline-binding region of S138A hPfn1 (H134S/S138A hPfn1), and by formin inhibition (Figure 5, F and G). Interestingly, abolishing both direct MT binding and formin interaction (G118V/S138A hPfn1 + SMIFH2 treatment) showed a tendency for a cumulative negative effect on axonal growth (Figure 5, F and G). In summary, our data show that the capacity of specific Pfn1 residues to mediate MT invasion of growth cone filopodia (even more than their ability to enhance MT growth speed) correlates with their effect in the regulation of axonal growth.

In vivo delivery of S138A hPfn1 efficiently promotes regeneration of peripheral and CNS axons. In the adult CNS, following the establishment of connections, axons mostly fail to regenerate after injury or disease. Our data demonstrate that S138A hPfn1 is a potent pro-regenerative molecule, capable of enhancing axonal growth in vitro both under permissive and inhibitory conditions. As a proof of concept, to further reveal its regenerative potential, we delivered S138A hPfn1 and the poly-proline-binding-deficient mutant $\mathrm{H} 134 \mathrm{~S} / \mathrm{S} 138 \mathrm{~A}$ hPfn1 in mice before either sciatic nerve injury or SCI. In order to easily trace Pfn1-expressing axons in vivo, we generated bicistronic expression vectors encoding enhanced GFP linked to S138A hPfn1 via the 2A self-cleaving small peptide (P2A), and packaged them into adeno-associated viral (AAV) particles containing the PHP.eB capsid, which allow noninvasive gene delivery to the nervous system (42). CAD cell extracts show that cells transfected with pAAV.GFP.P2A.S138A hPfn1 and pAAV.GFP. P2A.H134S/S138A hPfn1 plasmids present similar levels of overexpressed mutant Pfn1 (Supplemental Figure 3, A and B). In vivo, 2 weeks following viral administration through the tail vein, GFP expression was clearly detected throughout mouse brain, DRG, and spinal cord neurons (Supplemental Figure 3, C-E). To evaluate the in vivo regenerative capacity of peripheral axons expressing S138A hPfn1 or H134S/S138A hPfn1, AAVs were injected in the tail vein and 15 days later, sciatic nerves were crushed at the thigh level, allowing us to persistently define the crush site (Figure 6A). Three days after injury, in mice where pAAV.GFP.P2A.S138A hPfn1 was delivered, peripheral sensory axons regenerated over significantly longer distances than those of controls, as assessed both by SCG10 staining (Figure 6, B and C) and by measuring the dis- tance of $\mathrm{GFP}^{+}$axons from the lesion border, in which case almost 2-fold longer axons were found (Figure 6D). In contrast, when H134S/S138A hPfn1, i.e., the constitutively active Pfn1 mutant lacking the ability to bind poly-proline-containing proteins was delivered, the robust regenerative effect of S138A hPfn1 was substantially reduced (Figure 6, B-D) and the distance of $\mathrm{GFP}^{+}$axons to the lesion border was indistinguishable from that of control AAV-GFP-expressing axons (Figure 6D). At 28 days after injury, increased levels of active Pfn1 improved NMJ maturation; NMJs from S138A hPfn1-expressing animals presented a structural volume and complexity similar to that of the uninjured control group (Figure 6, E-G). In contrast, in AAV-GFP-injected animals, NMJs were not yet fully matured (Figure 6, E-G). Accordingly, nerve conduction velocity was improved by the increased levels of active Pfn1 (Figure 6H). The most striking effect of S138A hPfn1 delivery after sciatic nerve injury was restoration of mechanical nociception as it became indistinguishable from uninjured controls, while it was still severely impaired in AAV-GFP-expressing animals (Figure 6I). Altogether, our data place constitutively active Pfn1 as an attractive target for therapeutic strategies to induce peripheral nerve regeneration and functional recovery.

To further emphasize the action of active Pfn1 as a pro-regenerative molecule promoting axonal regeneration in vivo, AAVmediated delivery of S138A hPfn1 was conducted using a severe model of CNS trauma, complete spinal cord transection. Two weeks following viral administration through the tail vein (day-14, Figure 6J), injury was performed (day 0, Figure 6J). Six weeks following SCI, GFP-expressing axons were traced within the lesion site (Figure 6K and Supplemental Figure 3F). In comparison with animals injected with control AAV-GFP, animals treated with AAVs carrying GFP.P2A.S138A hPfn1 showed a 2.4-fold increased number of $\mathrm{GFP}^{+}$axons with the ability to penetrate the glial scar (Figure 6, $\mathrm{L}$ and $\mathrm{M}$ ) that displayed a 1.6-fold increased mean distance of regrowth from the rostral lesion border (Figure $6 \mathrm{~N}$ ). Whereas in control AAV-GFP-injected animals only $3 \%$ of the axons regenerated over distances above $450 \mu \mathrm{m}$, in GFP.P2A.S138A hPfn1-treated mice nearly $25 \%$ of the axons were able to regrow long distances from the rostral lesion border (Figure 60). These observations support the notion that in vivo delivery of active Pfn1 enhances the ability of regenerating axons to penetrate and grow within the inhibitory glial scar environment, in accordance with in vitro neurite outgrowth experiments in a nonpermissive substrate (Figure $4 \mathrm{~N}$ ). Combined, our findings indicate that increasing Pfn1 levels and activity enhance axonal regeneration both in high- and low-regenerative contexts, and hence identify Pfn1 as a therapeutic target to promote axonal regeneration upon injury.

\section{Discussion}

CNS regeneration is largely abortive in higher vertebrates because the plastic embryonic mechanisms underlying axonal growth are not reactivated following injury or disease. Damaged axons must assemble motile growth cones to restore functional deficits after trauma. This is likely dictated by the coordinated interplay between cytoskeletal components (43). The mechanical forces resulting from actin polymerization beneath the protruding membrane of the growth cone assign actin dynamics a fundamental role for growth cone motility, extension rate, and direction of axonal 

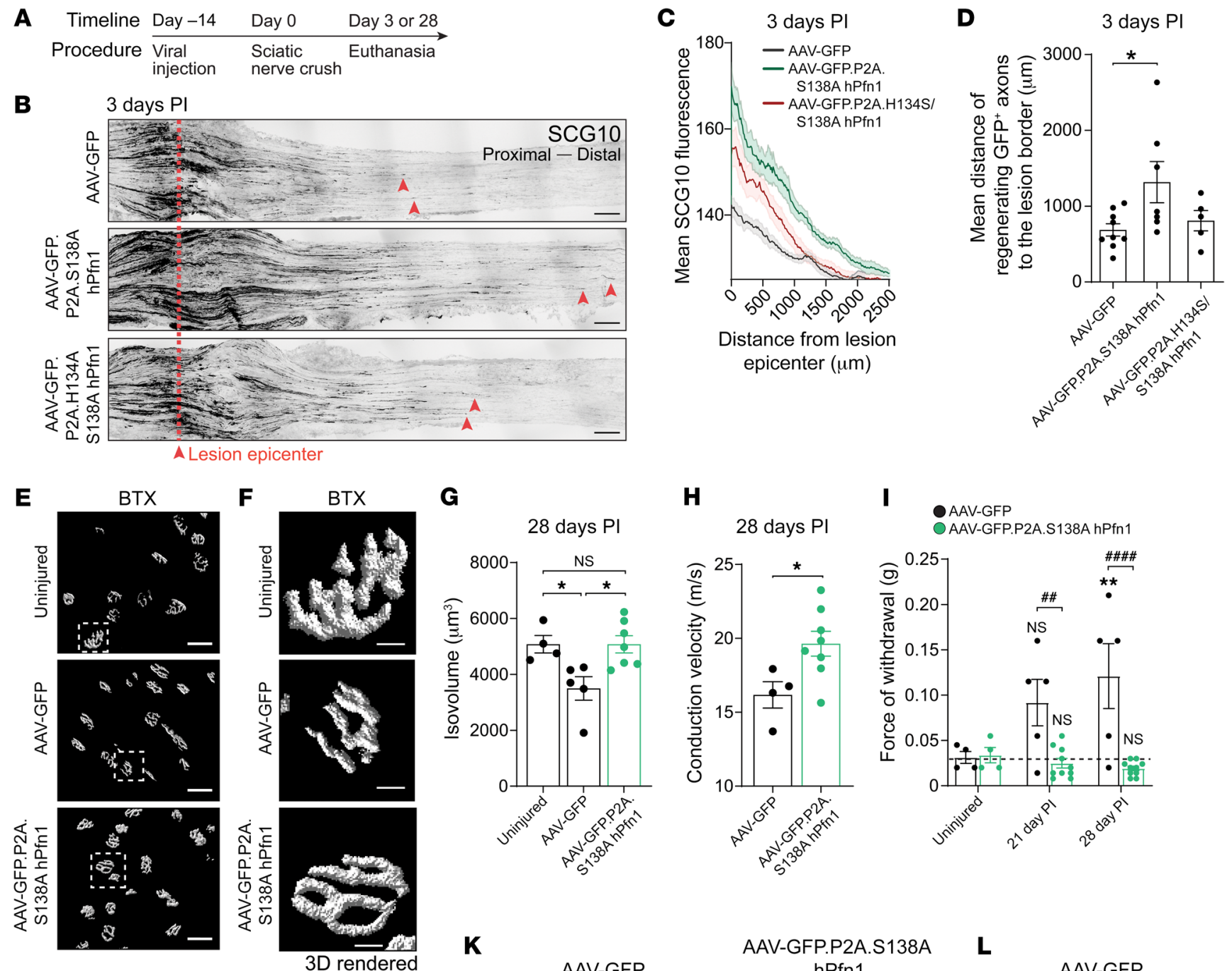

H
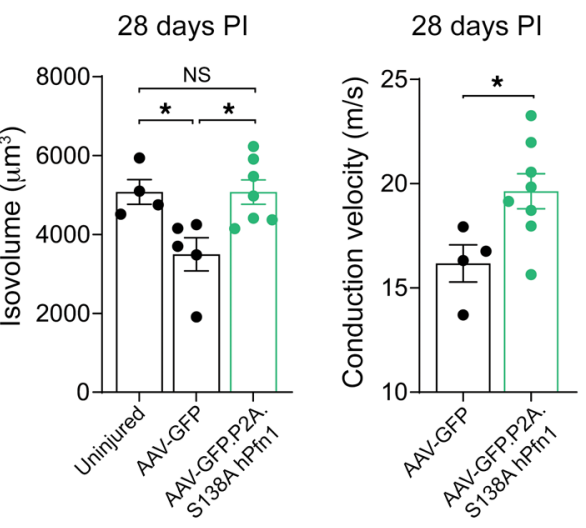

AAV-GFP.P2A.S138A hPfn1

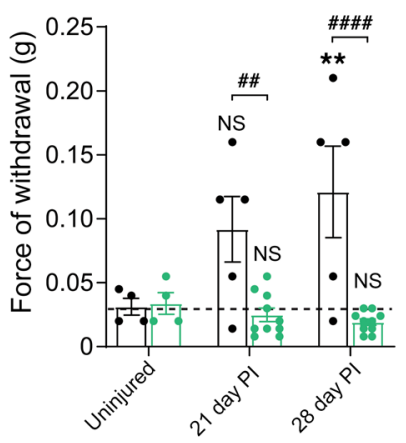

K

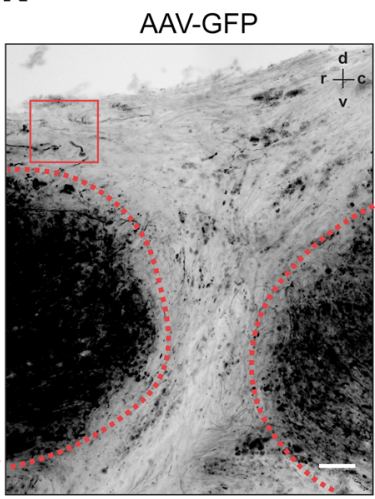

AAV-GFP.P2A.S138A hPfn1
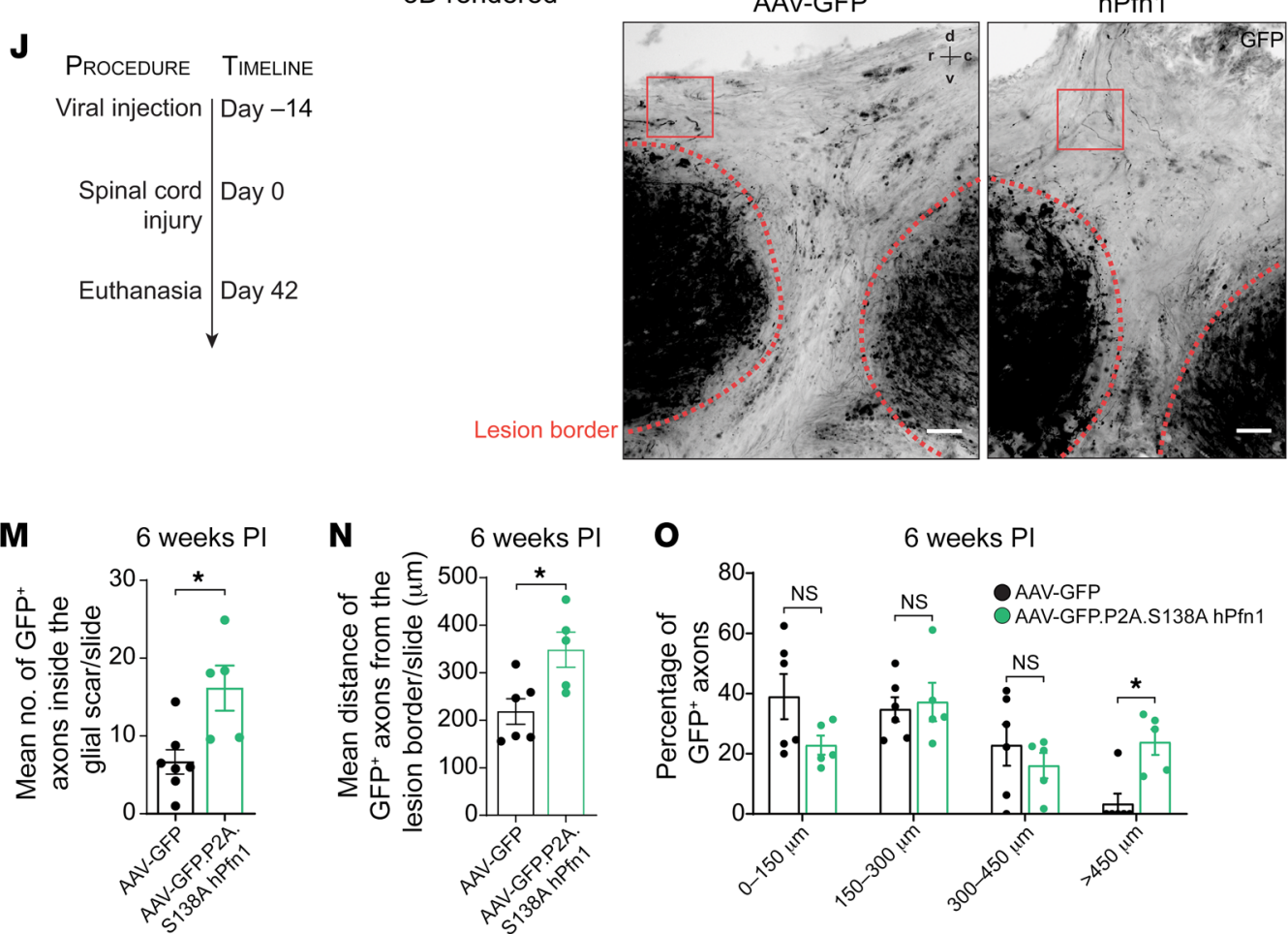

Lesion border

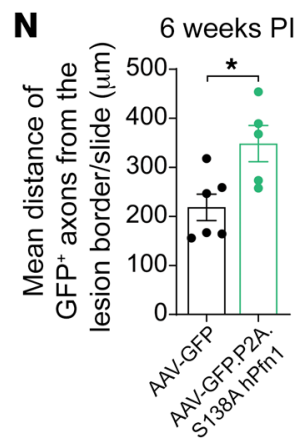

6 weeks $P$

o

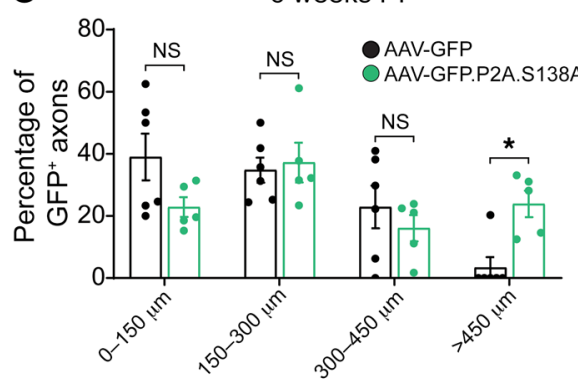

$\mathbf{L}$

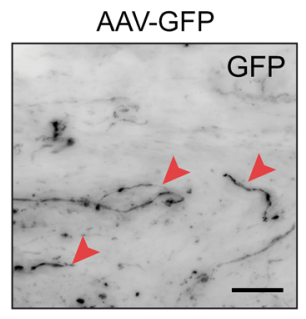

AAV-GFP.P2A.S138A hPfn1

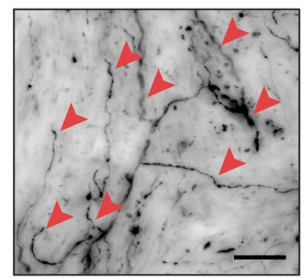


Figure 6. In vivo delivery of S138A hPfn1 elicits regeneration of peripheral and CNS axons. (A) Strategy to assess peripheral regeneration following viral delivery of S138A hPfn1. (B) SCG10 staining of longitudinal sciatic nerve sections at 3 days postinjury ( $\mathrm{PI}$ ); red dashed lines indicate the lesion epicenter, red arrowheads highlight regenerating axons. Scale bars: 200 $\mu \mathrm{m}$. (C) SCG10 fluorescence versus distance to lesion epicenter. (D) Mean distance of GFP+ sciatic nerve axons regenerating distally to the lesion edge 3 days PI. Data represent mean \pm SEM ( $n=5-9$ animals/condition). ${ }^{*} P<0.05$ by 1 -way ANOVA with Tukey's post hoc test. (E) 3D surface-rendered reconstructions, (F) zoom-in of $\mathbf{E}$, and (G) volume quantification of NMJs fluorescently labeled with $\alpha$-bungarotoxin (BTX), 28 days PI. Scale bars: $50 \mu \mathrm{m}(\mathbf{E})$ and $10 \mu \mathrm{m}(\mathbf{F})$. (H) Motor nerve conduction velocity, 28 days $\mathrm{PI}$. In $\mathbf{G}$ and $\mathbf{H}$, data represent mean \pm SEM ( $n=4-8$ animals/condition). ${ }^{*} P<0.05$ by Student's $t$ test. NS, not significant. (I) von Frey hair test, 21 and 28 days PI. Data represent mean \pm SEM ( $n=5-10$ animals/condition). ${ }^{*} P<0.01$; NS, not significant related to AAV-GFP uninjured condition. $\# \# P<0.01, \# \# \# P<0.0001$ refers to AAV-GFP versus AAV-GFP.P2A.S138A hPfn1 animals; both by 2-way ANOVA with Sidak's post hoc test. (J) Strategy to assess CNS regeneration following delivery of AAV-GFP and AAV-GFP. P2A.S138A hPfn1. (K) Injured spinal cords 6 weeks following transection. Scale bars: $100 \mu \mathrm{m}$. Red dashed line, lesion border; arrowheads, GFP+ axons within the lesion core; r, rostral; c, caudal; d, dorsal; v, ventral. (L) Zoom-ins of K. Scale bars: $40 \mu \mathrm{m}$. (M) Number of GFP+ axons regenerating within the glial scar. (N) Distance (rostral to caudal) of regenerating axons and (O) percentage of $\mathrm{CFP}^{+}$axons at different distance ranges from the injury border. Data represent mean \pm SEM $\left(n=5-7\right.$ animals/condition). ${ }^{*} P<0.05$ by Student's $t$ test. NS, not significant.

growth (44). Of note, actin and MT dynamics are intimately associated through cross-linkers, which help guide MTs toward preferential locations at peripheral growth cone edges (45). Interestingly, early in development, when neuronal symmetry is broken due to local protrusive events against the membrane, $\mathrm{Cfl}$ directs neurite formation by controlling actin retrograde dynamics and generating space for MT protrusion (15). Similarly, additional proteins regulating actin dynamics at the growth cone may play important roles for the cross-talk between actin and MTs, thereby strengthening the axon (re)growth potential. Here we identify Pfn1 as one such protein. In addition to the actin polymerization-promoting activity in growth cones, we show that neuronal Pfn1 profoundly modulates MT dynamics by supporting accelerated growth rates in axonal tips and by localizing growing MTs to growth cone filopodia. Enhanced non-muscle myosin II-based actin retrograde flow is generally viewed to slow down the rate of growth cone advance (46-48). Here, similarly to the results of Tedeschi et al. (19), we show that in conditions of optimal axonal regrowth such as those generated by $\mathrm{CL}$, actin retrograde is increased. Of note, actin retrograde flow can sweep MTs backwards in growth cones (49-51). In contrast, we show that increased actin retrograde flow can occur concomitantly with increased MT protrusion into growth cone filopodia. Interestingly, dynein is capable of enabling MTs to overcome non-muscle myosin II-driven forces, allowing their advance into growth cone filopodia, opposing axonal retraction (52). Putative molecular players allowing fast MT advance powered by $P f n 1$ in growth cones, in conditions of increased actin retrograde flow, should be further investigated.

Despite their similarities, Pfn1 and Pfn2 have different binding partners (53). This different ligand specificity can underlie distinct molecular functions. In fact, Pfn2 hinders neuritogenesis (54), supporting the specific effect of Pfn1 described here. Of note, whereas tubulin can be captured from a brain tissue extract on a Pfn1 column (53), it is absent when using Pfn2 affinity chromatography (53). In fact, our data support the idea that Pfn1, but probably not Pfn2, is a molecular linker of the actin and MT cytoskeletons. Additional in vitro assays using isolated proteins showed that tubulin and Pfn1 could be coimmunoprecipitated (26). More recently, using TIRF analysis in in vitro systems Pfn1 was shown to bind directly to MTs (25). Here we demonstrate that in the growth cone of cultured primary neurons, Pfn1 interferes both with MT growth speed and with MT invasion of filopodia. Whereas the effect of Pfn1 on MT growth speed is not secured by direct tubulin binding but by a positive cooperation with formins, its effect on localizing MTs to growth cone filopodia requires both direct MT binding and formins. Formins bind directly to MTs generally through the actin-related $\mathrm{FH} 2$ domain (36). Alternatively, formins can also associate with the MT plus-tip protein EB1 and thereby accumulate at MT plus ends, from where they nucleate and accelerate actin polymerization (55). Given that formins are only capable of potentiating actin elongation in the presence of Pfn, formin-Pfn complexes are probably important players in mediating the communication of the MT and actin cytoskeletons in growth cones. Indeed, peripheral dynamic MTs are deeply influenced by actin movements, as MTs are physically coupled to actin retrograde flow in the vertebrate growth cone periphery and exhibit similar rates of backward transport (56). Pfn1 is therefore perfectly suited to act as a molecular sensor coordinating the distribution of actin and MTs from a finite pool of basic units to distinct cytoskeletal networks.

In summary, we show that Pfn1 acts as a key coordinator of both actin and MT cytoskeletons in growth cones and thereby promotes axonal growth and regenerative capacity. Most importantly, we demonstrate that in vivo viral delivery of active Pfn1 promotes axonal regeneration and functional recovery of the injured sciatic nerve, and increases axonal regeneration through the inhibitory glial scar after SCI. Our results indicate that modulation of Pfn1 levels and activity is instrumental to successfully produce a positive regeneration outcome. Of note, AAVs are emerging as very attractive vehicles for clinical gene therapy of human nervous system disorders, given their low immunogenicity and toxicity and the ability of specific serotypes to cross the blood-brain barrier after intravenous delivery (42). In the future, AAV-mediated delivery of constitutively active Pfn1, together with the identification of modulators of Pfn1 activity with therapeutic potential, should be considered for the treatment of the injured nervous system.

\section{Methods}

Animals. Pfn1 neuron-specific conditional knockout mice (cre+ $\left.\mathrm{Pfn} 1^{\mathrm{f} / \mathrm{I}}\right)$ were generated by crossing homozygous Pfn1-floxed mice ( $\mathrm{Pfn} 1^{\mathrm{t} / \mathrm{f}}$; ref. 57) and single-neuron labeling with inducible Cre-mediated knockout (SLICK)-H mice (58). SLICK-H coexpress tamoxifen-inducible CreER ${ }^{\mathrm{T} 2}$ recombinase and YFP under the neuron-specific Thy1 promoter. Cre $\mathrm{Pfn} 1^{\mathrm{t} / \mathrm{wt}}$ mice were crossed with $\mathrm{Pfn} 1^{\mathrm{t} / \mathrm{wt}}$ mice such that $\mathrm{cre}^{+} \mathrm{Pfn} 1^{\mathrm{I} / \mathrm{fl}}$ and $\mathrm{cre}^{+} \mathrm{Pfn} 1^{\mathrm{w} / \mathrm{wt}}$ mice were generated. Genotyping was as described previously (57). Cre recombinase was induced by tamoxifen injection ( $75 \mathrm{mg} / \mathrm{kg}$; Sigma-Aldrich) at weaning for 5 days. Given tamoxifen's neuroprotective effects, controls were tamoxifen-treated $\mathrm{cre}^{+} \mathrm{Pfn} 1^{\mathrm{wt} / \mathrm{wt}}$ mice. Mice of either sex were used in all cre ${ }^{+} \mathrm{Pfn} 1$ pro- 
cedures. For AAV-mediated delivery of hPfn1, C57BL/6 mice (12-15 weeks old) were used; for SCI experiments, only females were used, whereas both sexes were used for sciatic nerve crush. Experimental conditions were randomized and surgeries were performed blinded to experimental conditions.

Primary cultures. DRG neuron cultures were performed as described previously (59). DRG from 7- to 8-week-old cre ${ }^{+} \mathrm{Pfn} 1$ mice or 6- to 8-week-old Wistar rats were used. For experiments in which DRG were conditioned, sciatic nerve transection was done 1 week prior to culture. Electroporation of DRG neurons was performed with a 4D-Nucleofector System (mouse DRG neurons, program CM-137; rat DRG neurons, program CM-138) at a cell density of at least 200,000 cells/condition and left in suspension for 24 hours at $37^{\circ} \mathrm{C}$ in $5 \% \mathrm{CO}_{2}$. Subsequently, cells were grown on $13-\mathrm{mm}$ coverslips (for neurite outgrowth assays) or 8-well $\mu$-dishes (IBIDI-80827, for live imaging assays) coated with poly-L-lysine (PLL) $(20 \mu \mathrm{g} / \mathrm{mL}$, Sigma-Aldrich, P2636) and laminin (5 $\mu \mathrm{g} / \mathrm{mL}$, Sigma-Aldrich, L2020) for 12 to 14 hours until fixing or imaging. For experiments using aggrecan (Sigma-Aldrich, A1960-1MG), DRG neurons were plated in either PLL/laminin (20:5 $\mu \mathrm{g} / \mathrm{mL})$ or PLL/laminin/aggrecan (20:5:20 $\mu \mathrm{g} / \mathrm{mL}$ ). Culture medium was DMEM/F12 (Sigma-Aldrich, D8437) supplemented with 1× B27 (Gibco), 1\% penicillin/streptomycin (Gibco), $2 \mathrm{mM} \mathrm{L-glutamine} \mathrm{(Gibco),} \mathrm{and} 50 \mathrm{ng} / \mathrm{mL} \mathrm{NGF} \mathrm{(Millipore,}$ 01-125). Hippocampal neurons were cultured as described previously (60). Electroporation was performed at a density of at least 750,000 cells/condition (program CU110). Cells were plated either in 13-mm coverslips or 8 -well $\mu$-dishes coated with PLL $(20 \mu \mathrm{g} / \mathrm{mL})$ grown in Neurobasal medium supplemented with $1 \%$ penicillin-streptomicin, $1 \times \mathrm{B} 27,2 \mathrm{mM}$ L-glutamine, and maintained at $37^{\circ} \mathrm{C}$ in $5 \% \mathrm{CO}_{2}$. Hippocampal neurons at DIV4 (for axonal growth analysis) or DIV7 (for dendritic growth analysis) and DRG neurons (12 to 14 hours after plating) were fixed for immunostaining or imaged for live-cell experiments, as detailed below.

Plasmids and viral vectors. The full-length human Pfn1 open reading frame (WT hPfn1, cloned in the pCMV-SPORT6 vector, Addgene, clone IRATp970C034D) and different hPfn1 mutants were used. Specific WT hPfn1 residues were mutated to generate an shRNA-resistant WT hPfn1 (WT hPfn1*), phosphorylation-resistant constitutively active hPfn1 (S138A hPfn1), poly-proline-binding-deficient hPfn1 (H134S hPfn1), and MT binding-deficient hPfn1 (G118V hPfn1). Mutants were obtained using the QuickChange II XL kit (Agilent Technologies) and mismatched primers introducing 1- or 2-bp substitutions. Pfn1 (target sequence: CGGTGGTTTGATCAACAAGAA, TRCN0000011969, Sigma-Aldrich) and Pfn2 (target sequence: ACGTTGATGGTGACTGCACAA, TRCN0000071642, Sigma-Aldrich) shRNA constructs were used in hippocampal and DRG neuron cultures and in CAD cells (European Collection of Authenticated Cell Cultures, 08100805) to downregulate Pfn1 and Pfn2 protein levels, respectively. For the in vivo delivery of S138A hPfn1 and H134S/S138A hPfn1, AAVs were used. In detail, GFP linked to S138A hPfn1 by the 2A self-cleaving small peptide P2A was subcloned into an AAV-PHP.eB plasmid to obtain the constructs pAAV-GFP.P2A.S138A hPfn1 and pAAV-GFP.P2A.H134S/S138A hPfn1. Control AAV vectors, in which Pfn1 was replaced by a 5-glycine sequence (pAAV-GFP), were also generated. The viral vectors are herein referred to as AAV-GFP, AAV-GFP.P2A.S138A hPfn1, or AAV-GFP. P2A.H134S/S138A hPfn1. Expression was driven by the neuronal synapsin promoter. AAV-PHP.eB particles were produced by Vector Builder and enabled neuronal-specific targeting of the nervous system following systemic delivery (42).

Neurite outgrowth and growth cone morphology. Neurite outgrowth was assessed following immunofluorescence with antibodies against $\beta$ III-tubulin. DRG neuron cultures of $\mathrm{cre}^{+} \mathrm{Pfn} 1^{\mathrm{fl} / \mathrm{fl}}$ and $\mathrm{cre}^{+} \mathrm{Pfn} 1^{\mathrm{wt} / \mathrm{wt}}$ mice were fixed 12 to 14 hours after plating with $4 \%$ paraformaldehyde (PFA). Incubation with mouse anti- $\beta$ III-tubulin (1:1,000; Promega, G7121) was done overnight at $4^{\circ} \mathrm{C}$. The secondary antibody was donkey anti-mouse-Alexa Fluor 594 (1:1,000; Jackson ImmunoResearch Labs, 715-585-150). Images were acquired by epifluorescence in a Zeiss Axio Imager Z1 microscope with an Axiocam MR3.0 camera and Axiovision 4.7 software. Neurite tracing and branching analyses were performed in Matlab with Synapse Detector software (61) to quantify neurites crossing concentric circles centered at the cell body with radiuses of consecutive multiples of $25 \mu \mathrm{m}$. In experiments in which WT hPfn1 or S138A hPfn1 overexpression was performed, dissociated adult rat DRG neurons were electroporated (as detailed above) with a mixture of plasmids encoding GFP $(0.2 \mu \mathrm{g}$, pmaxGFP, Lonza) and hPfn1 $(0.6 \mu \mathrm{g})$, plated at a density of 7,500 cells/well, fixed 12 to 14 hours after plating, and stained for $\beta$ III-tubulin as described above. Image acquisition was performed using a Leica DMI 6000B with an ORCA-Flash4.0 V2 C11440-22CU digital camera and Leica Application Suite Advanced Fluorescence (LAS AF) software. Experiments using shRNA constructs followed similar procedures. Specifically, mixtures of pmaxGFP/Pfn1 shRNA (0.2:1.2 $\mu \mathrm{g})$ or Discosoma $s p$. red fluorescent protein (DsRed, Clontech)/shRNA Pfn2-encoding plasmids $(0.5: 1.5 \mu \mathrm{g})$ were used; control experimental conditions were nucleofected with the empty plasmid pLKO.1 (CTR, Addgene). E18 rat hippocampal neurons were electroporated following the same strategy and fixed on DIV4 or DIV7. For Pfn1-depleted hippocampal neurons, axonal and dendritic lengths were traced manually with the NeuronJ plugin for ImageJ (Fiji). Axonal tracing was also performed in DIV4 hippocampal neurons cotransfected with pmaxGFP $(0.2 \mu \mathrm{g})$ and S138A hPfn1, G118V/S138A hPfn1, or H134S/S138A hPfn1 (0.6 $\mu \mathrm{g})$. Polarization analysis of hippocampal neurons was assessed as detailed previously (15). Morphometric evaluation of growth cones was performed in both $\mathrm{cre}^{+} \mathrm{Pfn} 1^{\mathrm{t} / \mathrm{l} l}$ and $\mathrm{cre}^{+} \mathrm{Pfn} 1^{\mathrm{wt} / \mathrm{wt}}$ adult $\mathrm{DRG}$ neurons and hippocampal neurons expressing shRNA Pfn2 plasmid. Neurons were stained with mouse anti- $\beta$ III-tubulin $(1: 5,000)$ overnight at $4^{\circ} \mathrm{C}$ and incubated for 1 hour at room temperature with donkey anti-mouseAlexa Fluor 647 (1:500; Jackson ImmunoResearch Labs, 715-605-150) secondary antibody and with rhodamine-conjugated phalloidin (1:50; Thermo Fisher Scientific, R415) diluted 1:10 in blocking buffer. Images were acquired by epifluorescence on a Zeiss Axio Imager Z1 microscope with an Axiocam MR3.0 camera and Axiovision 4.7 software. Only growth cones of $\mathrm{YFP}^{+} / \beta \mathrm{III}$-tubulin ${ }^{+}$(in the case of DRG neurons from cre ${ }^{+} \mathrm{Pfn} 1$ mice) and $\mathrm{GFP}^{+}$neurons (in the case of hippocampal neurons conucleofected with pmaxGFP) were analyzed by measuring the total area of the growth cone, and the filopodia number and size using ImageJ software. To quantify endogenous Pfn1 fluorescence in naive and conditioned DRG growth cones, neurons were fixed 12 to 14 hours after plating with $2 \%$ PFA, stained with rabbit anti-Pfn1 (1:400; Abcam, ab50667) overnight at $4^{\circ} \mathrm{C}$, and incubated for 1 hour at room temperature with donkey anti-mouse Alexa Fluor 594 (1:1,000) and goat anti-rabbit Alexa Fluor 488 (1:1,000; Jackson ImmunoResearch Labs, 111-545-003) secondary antibodies in blocking buffer. Images were acquired by epifluorescence as described above. A line scan 
across growth cones was drawn, a plot of gray values was done in relation to the distance from the growth cone leading edge, and corresponding values were extracted and compared (for each image the highest gray value was considered $100 \%$ ).

Live-cell imaging. For the analysis of actin and MT dynamics in the growth cone, adult DRG neurons from 7- to 8-week-old cre ${ }^{+} \mathrm{Pfn} 1^{1 / 1 / 1}$ and $\mathrm{cre}^{+} \mathrm{Pfn} 1^{\mathrm{wt} / \mathrm{wt}}$ mice were isolated as described above and nucleofected with plasmids encoding LifeAct-RFP (62) $(0.75 \mu \mathrm{g})$ or EB3-mCherry (0.5 $\mu$ g; ref. 63), respectively. Twelve hours after plating, time-lapse recordings were performed in phenol-free DMEM/F12 supplemented as mentioned above, at $37^{\circ} \mathrm{C}$ and $5 \% \mathrm{CO}_{2}$, on a Spinning Disk Confocal System Andor Revolution XD with an iXonEM+ DU-897 camera and a IQ1.10.1 software (ANDOR Technology). Only transfected RFP ${ }^{+} / \mathrm{YFP}^{+}$ or $\mathrm{mCherry}^{+} / \mathrm{YFP}^{+}$neurons were considered for analysis. For both the quantification of actin retrograde flow and of EB3 comet growth speed, kymographs were performed using the Fiji KymoResliceWide plugin (distance, $x$ axis; time, $y$ axis). Starting and end positions of the traces were defined using the Fiji Cell Counter plugin. In live imaging experiments using hippocampal or DRG neurons from Wistar rats, plasmids expressing either LifeAct-GFP (0.75 $\mu \mathrm{g})(62)$ or EB3-GFP (0.5 $\mu \mathrm{g})(64)$ were conucleofected alongside plasmids of interest. Actin retrograde flow and EB3 comet speed were quantified in these neurons, as well as the EB3 comet invasion per filopodia. The invasion frequency of dynamic MTs was quantified by counting the number of EB3 comet invasions (using the Fiji plugin Cell Counter) divided by the number of filopodia. A similar approach was used in SMIFH2-treated $(5 \mu \mathrm{M}$, Sigma-Aldrich, S4826) cells. SMIFH2 is a general formin inhibitor that targets diverse formin isoforms (38), decreasing their affinity for the barbed end of actin filaments, preventing both actin nucleation and processive barbed-end elongation. In SMIFH2 experiments, hippocampal neurons underwent 2 drug treatments, on DIV3 and at DIV4, 1 hour before fixing.

Immunoblotting. Protein lysates of rat SCI sites (collected $2.5 \mathrm{~mm}$ rostral and $2.5 \mathrm{~mm}$ caudal to the lesion site of animals with either SCI or CL 1 week following injury), DRG, brain (from $\mathrm{cre}^{+} \mathrm{Pfn} 1^{\mathrm{t} / \mathrm{fl}}$ and $\mathrm{cre}^{+}$ $\mathrm{Pfn}^{\mathrm{wt} / \mathrm{wt}}$ mice), or CAD cell extracts were prepared in ice-cold lysis buffer containing 0.3\% Triton X-100 (MilliporeSigma), protease inhibitors (cOmplete, Mini; Roche), and $2 \mathrm{mM}$ orthovanadate, separated under denaturing conditions, transferred to Amersham Protran Premium $0.45-\mu \mathrm{m}$ nitrocellulose membranes (GE Healthcare Life Sciences), and probed with primary antibodies (in $5 \%$ BSA or $5 \%$ milk in TBS-T) overnight at $4^{\circ} \mathrm{C}$. Primary antibodies used were rabbit anti-Pfn1 (1:1,000; either Thermo Fisher Scientific, PA5-17444 or Abcam, ab50667), rabbit anti-Pfn1 p-S138 (1:1,000, provided by Jieya Shao, UCSF, San Francisco, California, USA), rabbit anti-Pfn2 (1:1,000, provided by Pietro Pilo Boyl, Institute of Genetics University of Bonn, Germany), mouse anti- $\beta$-actin (1:5,000; Sigma-Aldrich, A5441), mouse anti- $\alpha$-tubulin (1:1,000; Sigma-Aldrich, T6199), rabbit anti-HPRT (1:1,000; Santa Cruz Biotechnology, sc-20975), rabbit anti-ROCK1 (1:1,000, Abcam, ab134181, clone EPR638Y), and rabbit anti-vinculin (1:1,000; Abcam, ab129002). Secondary antibodies were used in 5\% nonfat dried milk in TBS-T for 1 hour at room temperature: donkey anti-mouse IgG conjugated with horseradish peroxidase (HRP) (1:5,000; Jackson ImmunoResearch Labs, 715-035-151) and donkey anti-rabbit IgG conjugated with HRP (1:5,000; Jackson ImmunoResearch Labs, 711-035-152). Membranes were incubated with Luminata Crescendo Western HRP substrate (Millipore), exposed to Fuji Medical X-Ray Film (Fujifilm), scanned on a Molecular Imager GS800, and quantified using Quantity One 1-D Analysis Software version 4.6 (Bio-Rad).

Analysis of peripheral axon regeneration and functional recovery. Adult 12-week-old mice were deeply anesthetized with isoflurane. Sciatic nerves were crushed at the mid-thigh level for 15 seconds, 2 consecutive times, using hemostatic forceps (13010-12, FST), producing a well-defined lesion area. For histological analysis of axonal regeneration, remyelination, and NMJ establishment in cre ${ }^{+} \mathrm{Pfn} 1$ mice, animals were sacrificed at 7, 15, or 28 days after injury. To analyze regenerated myelinated sciatic nerve axons and g-ratio, nerves were collected at the same anatomical position distal to the crush site, just above the bifurcation of the sciatic nerve, fixed in $4 \%$ glutaraldehyde in $0.1 \mathrm{M}$ cacodylate buffer ( $\mathrm{pH}$ 7.4) for 1 week, postfixed with $1 \% \mathrm{OsO}_{4}$ in 0.1 M sodium cacodylate buffer ( $\mathrm{pH}$ 7.4) for 2 hours, and dehydrated and embedded in Epon (Electron Microscopy Sciences). Sections (1 $\mu \mathrm{m}$ thickness) were stained for 10 minutes with $1 \% p$-phenylenediamine (PPD) in absolute methanol, dried, and mounted on DPX (Merck). Images of the lesion area were acquired using an Olympus optical microscope with an Olympus DP 25 camera and analyzed in Photoshop (Adobe). The total number of myelinated axons was determined in each cross section and divided by its area. Analysis of unmyelinated axons was not performed in cre ${ }^{+} \mathrm{Pfn} 1$ mice, as Cre expression only occurs in a small percentage of small, unmyelinated neurons. The g-ratio was calculated by dividing the diameter of each axon by its myelin-including diameter in over 50 axons per animal. For the morphometric evaluation of NMJs, the lateral gastrocnemius was dissected in PBS under a stereomicroscope, and fixed for 24 hour with $4 \% \mathrm{PFA}$ at $4^{\circ} \mathrm{C}$. Isolated muscles were permeabilized for 30 minutes with $1 \%$ Triton $\mathrm{X}-100$ at room temperature, and the autofluorescence quenched with $0.2 \mathrm{M} \mathrm{NH}_{4} \mathrm{Cl}$ (Merck) and $0.1 \%$ sodium borohydride (MilliporeSigma). After 1 hour of blocking ( $1 \mathrm{mg} / \mathrm{mL} \mathrm{BSA,} 0.2 \%$ Triton $\mathrm{X}-100)$, tissues were incubated 1 hour with rhodamine-conjugated $\alpha$-bungarotoxin (1:250, Thermo Fischer Scientific, T1175) in blocking buffer at room temperature. NMJ morphometric analysis was carried out in $Z$-stack images taken with a Leica TCS SP8 microscope, 3D-rendered using Huygens Professional software (Scientific Volume Imaging, SVI), and analyzed for volume. Analysis of motor nerve conduction velocity was performed as described previously (65) with a PowerLab 4/25T (AD Instruments) using Chart5 software. Conduction velocities were calculated as (proximal distance - distal distance)/(proximal latency - distal latency). To assess regeneration of sciatic nerve axons following AAV-PHP.eB-mediated delivery of S138A hPfn1 or H134S/ S138A hPfn1, the sciatic nerve was crushed 2 weeks after systemically injecting AAV-GFP, AAV-GFP.P2A.S138A hPfn1, or AAV-GFP.P2A. H134S/S138A hPfn1 $\left(6 \times 10^{11} \mathrm{vg} /\right.$ mouse $)$ through the tail vein, using the AAV-PHP.eB capsid that allows noninvasive gene delivery to the nervous system (42). Mice recovered for 3 or 28 days before sacrifice. Nerves were collected after $4 \%$ PFA perfusion, postfixed for 3 days at $4^{\circ} \mathrm{C}$, and cryoprotected in $30 \%$ sucrose. Tissues were embedded in optimum cutting temperature compound (Thermo Fisher Scientific), frozen, and sectioned longitudinally (Leica) at $15 \mu \mathrm{m}$ thickness. In these nerves, SCG10 expression was detected by immunofluorescence using rabbit anti-SCG10/stathmin-2 (1:10,000; Novus, NBP149461). Image acquisition was performed using an IN Cell Analyzer 2000 (GE Healthcare) and analyzed using Fiji software. Quantification of SCG10 fluorescence was performed in longitudinal sections by scanning a thickness similar to that of the nerve sample. A plot of 
mean gray values was done in relation to the distance of the lesion epicenter. Axonal regeneration was additionally quantified distally to the injury site by measuring the distance from the distal tip of $\mathrm{GFP}^{+}$ regenerating axons to the lesion border (up to 4 sections per animal were analyzed). Data represent the mean distance for each condition, considering all regenerating axons. Accumulation of nuclei of inflammatory cells within the crush site was visualized after DAPI counterstaining and used to define the lesion area. Regenerating axons were seen as continuous structures that could be clearly separated from degenerating swollen axonal fragments under high magnification. Analysis of NMJs and motor nerve conduction velocity was conducted as described above. For von Frey hair testing, animals were acclimatized for 20 minutes in a chamber with a wire-mesh bottom allowing access to hind paws. Retractable monofilaments (Aesthesio, Precise Tactile Sensory Evaluator, 37450-275) were used to apply a force to the mid-plantar surface on hind paws. Clear paw withdrawal or abrupt moving were considered positive responses. Withdrawal threshold equaled the weakest force to elicit paw withdrawal on $50 \%$ or more of the trials ( $n=5$ trials). The percentage of the withdrawal threshold shown is an averaged value of right and left hind paws relative to baseline recordings done in uninjured AAV-GFP-injected animals.

Analysis of axonal regeneration following SCI. Adult 8-week-old $\mathrm{cre}^{+} \mathrm{Pfn} 1$ mice and 15-week-old female C57BL/6 mice were deeply anesthetized with isoflurane. Laminectomy was performed at the thoracic T8 to T9 level and the spinal cord was cut using a micro feather ophthalmic scalpel (Feather, Safety Razor Co). For CL experiments, animals were subjected to a sciatic nerve transection 1 week prior to SCI. In cre ${ }^{+} \mathrm{Pfn} 1$ mice, analysis of dorsal column axon regeneration after either SCI or CL was performed 4 weeks after injury. Dorsal column axons were traced by injecting $2 \mu \mathrm{L}$ of $1 \%$ CT-B (List Biologicals, 103B) with a 10- $\mu \mathrm{L}$ syringe (Hamilton) into the left sciatic nerve 4 days prior to euthanasia (day 24 after injury). On day 28, mice were perfused with $4 \%$ PFA and the spinal cords were postfixed for 1 week at $4^{\circ} \mathrm{C}$ and later cryoprotected in $30 \%$ sucrose. Serial tissue sagittal cryosections (50 $\mu \mathrm{m}$ thickness) were collected for free-floating immunohistochemistry. Sections were incubated overnight at $4^{\circ} \mathrm{C}$ with goat anti-CT-B primary antibody (1:30,000; List Biologicals, 703). Antigen detection was amplified by incubating samples with biotinylated horse anti-goat antibody (1:200; Vector) for 2 hours at room temperature and subsequently with Alexa Fluor 568-streptavidin (1:1,000, Invitrogen) for 1 hour at room temperature. Dorsal column fiber images were acquired by confocal microscopy on a Leica TCS SP5 II with LAS AF software and analyzed using Fiji software. Regeneration of dorsal column axons was quantified by measuring the distance from the rostral tip of all regenerating $\mathrm{YFP}^{+} / \mathrm{CT}-\mathrm{B}^{+}$axons to a vertical line placed at the rostral end of the dorsal column tract (up to 6 sections per animal). Data presented are the mean distance for each condition, considering all regenerating axons.

To assess axonal regeneration of spinal cord axons following AAV-PHP.eB-mediated delivery of Pfn1, spinal cord transection was performed 2 weeks after systemically injecting AAV-GFP, AAV-GFP. P2A.S138A hPfn1, or AAV-GFP.P2A.H134S/S138A hPfn1 $\left(4 \times 10^{11} \mathrm{vg} /\right.$ mouse) through the tail vein, using the AAV-PHP.eB capsid that allows noninvasive gene delivery to the nervous system (42). Injured spinal cord tissue was collected 6 weeks after SCI and processed as described above; image acquisition was performed using the IN Cell Analyzer 2000 microscope and analyzed using Fiji software. The total number of $\mathrm{GFP}^{+}$axons within the glial scar were counted in up to 12 sections per animal. Regenerating rostral to caudal distances were measured from the tip of $\mathrm{GFP}^{+}$axons to a vertical line placed perpendicularly to the sagittal axis of the spinal cord at the rostral border of the lesion. Regenerating distances are presented as the mean value considering all regenerating $\mathrm{GFP}^{+}$axons, and as a percentage of $\mathrm{GFP}^{+}$axons found within different growth distance windows $(0-150 \mu \mathrm{m}, 150-300 \mu \mathrm{m}$, 300-450, and $>450 \mu \mathrm{m})$.

Statistics. All statistical tests were performed with GraphPad Prism 6. Unless otherwise stated, the following statistical tests were used: 2-tailed Student's $t$ test, 1-way ANOVA with Tukey's post hoc test, and 2-way ANOVA with Sidak's and Tukey's post hoc test. A P value less than 0.05 was considered significant. Statistical tests and sample sizes are indicated in figure legends and significance was defined as ${ }^{*}$ or ${ }^{\#} P<0.05 ;{ }^{* *}$ or ${ }^{\# \#} P<0.01 ;{ }^{* * *} P<0.001 ;{ }^{* * * *}$ or ${ }^{\# \# \# \#} P<0.0001$; NS or \#N, not significant.

Study approval. Experiments were carried out in accordance with the European Union Directive 2010/63/EU and national Decreto-lei $\mathrm{n}^{0} 113-2013$. The protocols described were approved by the IBMC Ethical Committee and by the Portuguese Veterinarian Board.

\section{Author contributions}

MMS coordinated the research. MMS and RPC designed and analyzed the experiments and wrote the manuscript. RPC, SCS, SCL, JNR, TFS, DM, JM, ACC, PB, and MMS performed the experiments and quantifications. MAL and FB provided conceptual and experimental support. $\mathrm{MC}$ and $\mathrm{RF}$ provided $\mathrm{Pfn} 1^{\mathrm{fl} / \mathrm{fl}}$ mice. $\mathrm{FB}$, MAL, PB, and RF critically revised the manuscript.

\section{Acknowledgments}

The authors are indebted to F. Mar, R. Baeta, and D. Silva (Nerve Regeneration group) for experimental support. We thank the i3S Animal, Cell Culture, Genotyping, Histology and Electron Microscopy, Advanced Light Microscopy and BioSciences Screening (PPBI-POCI-01-0145-FEDER-022122) Facilities. We thank Marco Rust (Philipps-Universität Marburg, Germany) for transferring $P f n 1^{1 / / 1}$ mice. This work was financed by FEDER - Fundo Europeu de Desenvolvimento Regional funds through the NORTE 2020 Norte Portugal Regional Operational Programme, Portugal 2020, and by Portuguese funds through FCT - Fundação para a Ciência e a Tecnologia/Ministério da Ciência, Tecnologia e Ensino Superior in the framework of the project NORTE-01-0145-FEDER-028623 (PTDC/MED-NEU/28623/2017). Additional funding was from the International Foundation for Research in Paraplegia (P140), RESOLVE (NORTE-01-0246-FEDER-000018), the Morton Cure Paralyis Fund (MCPF), and The Bluepharma | University of Coimbra Innovation Award. RPC, SCS, JNR, and ACC are FCT fellows (SFRH/BD/112112/2015, SFRH/BD/136760/2018, SFRH/ BD/131565/2017 and SFRH/BD/143926/2019, respectively). ML is an FCT Investigator.

Address correspondence to: Mónica Mendes Sousa, Nerve Regeneration Group, Program in Neurobiology and Neurologic Disorders, Instituto de Biologia Molecular e Celular (IBMC) and Instituto de Inovação e Investigação em Saúde, Universidade do Porto, Rua Alfredo Allen 208, 4200-135 Porto, Portugal. Phone: 351.220.408.800; Email: msousa@ibmc.up.pt. 
1. Mar FM, Bonni A, Sousa MM. Cell intrinsic control of axon regeneration. EMBO Rep. 2014;15(3):254-263.

2. Nascimento AI, Mar FM, Sousa MM. The intriguing nature of dorsal root ganglion neurons: Linking structure with polarity and function. Prog Neurobiol. 2018;168:86-103.

3. Neumann S, Woolf CJ. Regeneration of dorsal column fibers into and beyond the lesion site following adult spinal cord injury. Neuron. 1999;23(1):83-91.

4. Blanquie O, Bradke F. Cytoskeleton dynamics in axon regeneration. Curr Opin Neurobiol. 2018;51:60-69.

5. Gomez TM, Letourneau PC. Actin dynamics in growth cone motility and navigation. J Neurochem. 2014;129(2):221-234.

6. Bradke F, Dotti CG. The role of local actin instability in axon formation. Science. 1999;283(5409):1931-1934.

7. Lowery LA, Van Vactor D. The trip of the tip: understanding the growth cone machinery. Nat Rev Mol Cell Biol. 2009;10(5):332-343.

8. Joset A, Dodd DA, Halegoua S, Schwab ME. Pincher-generated Nogo-A endosomes mediate growth cone collapse and retrograde signaling. JCell Biol. 2010;188(2):271-285.

9. Mimura F, et al. Myelin-associated glycoprotein inhibits microtubule assembly by a Rhokinase-dependent mechanism. J Biol Chem. 2006;281(23):15970-15979.

10. Monnier PP, Sierra A, Schwab JM, Henke-Fahle S, Mueller BK. The Rho/ROCK pathway mediates neurite growth-inhibitory activity associated with the chondroitin sulfate proteoglycans of the CNS glial scar. Mol Cell Neurosci. 2003;22(3):319-330.

11. Dergham P, Ellezam B, Essagian C, Avedissian H, Lubell WD, McKerracher L. Rho signaling pathway targeted to promote spinal cord repair. JNeurosci. 2002;22(15):6570-6577.

12. Lehmann $\mathrm{M}$, et al. Inactivation of Rho signaling pathway promotes CNS axon regeneration. J Neurosci. 1999;19(17):7537-7547.

13. Fehlings MG, et al. A phase I/IIa clinical trial of a recombinant Rho protein antagonist in acute spinal cord injury. J Neurotrauma. 2011;28(5):787-796.

14. Pavlov D, Muhlrad A, Cooper J, Wear M, Reisler E. Actin filament severing by cofilin. J Mol Biol. 2007;365(5):1350-1358.

15. Flynn KC, et al. ADF/cofilin-mediated actin retrograde flow directs neurite formation in the developing brain. Neuron. 2012;76(6):1091-1107.

16. Didry D, Carlier MF, Pantaloni D. Synergy between actin depolymerizing factor/cofilin and profilin in increasing actin filament turnover. J Biol Chem. 1998;273(40):25602-25611.

17. Garvalov BK, et al. Cdc42 regulates cofilin during the establishment of neuronal polarity. J Neurosci. 2007;27(48):13117-13129.

18. Wen Z, Han L, Bamburg JR, Shim S, Ming GL, Zheng JQ. BMP gradients steer nerve growth cones by a balancing act of LIM kinase and Slingshot phosphatase on ADF/cofilin. J Cell Biol. 2007;178(1):107-119.

19. Tedeschi A, et al. ADF/cofilin-mediated actin turnover promotes axon regeneration in the adult CNS. Neuron. 2019;103(6):1073-1085.e6.

20. Witke W. The role of profilin complexes in cell motility and other cellular processes. Trends Cell Biol. 2004;14(8):461-469.

21. Ferron F, Rebowski G, Lee SH, Dominguez R. Structural basis for the recruitment of profilinactin complexes during filament elongation by Ena/VASP. EMBO J. 2007;26(21):4597-4606.

22. Paul AS, Pollard TD. Review of the mechanism of processive actin filament elongation by formins. Cell Motil Cytoskeleton. 2009;66(8):606-617.

23. Michaelsen-Preusse K, et al. Neuronal profilins in health and disease: Relevance for spine plasticity and Fragile X syndrome. Proc Natl Acad Sci U S A. 2016;113(12):3365-3370.

24. Bender M, et al. Megakaryocyte-specific profilin1deficiency alters microtubule stability and causes a Wiskott-Aldrich syndrome-like platelet defect. Nat Commun. 2014;5:4746.

25. Henty-Ridilla JL, Juanes MA, Goode BL. Profilin directly promotes microtubule growth through residues mutated in amyotrophic lateral sclerosis. Curr Biol. 2017;27(22):3535-3543.e4.

26. Nejedla M, et al. Profilin connects actin assembly with microtubule dynamics. Mol Biol Cell. 2016;27(15):2381-2393.

27. Metzler WJ, Bell AJ, Ernst E, Lavoie TB, Mueller L. Identification of the poly-L-prolinebinding site on human profilin. J Biol Chem. 1994;269(6):4620-4625.

28. Lassing I, Lindberg U. Specific interaction between phosphatidylinositol 4,5-bisphosphate and profilactin. Nature. 1985;314(6010):472-474

29. Smith DS, Skene JH. A transcription-dependent switch controls competence of adult neurons for distinct modes of axon growth. J Neurosci. 1997;17(2):646-658

30. Shin JE, Geisler S, DiAntonio A. Dynamic regulation of SCG10 in regenerating axons after injury. Exp Neurol. 2014;252:1-11.

31. Shao J, Welch WJ, Diprospero NA, Diamond MI. Phosphorylation of profilin by ROCK1 regulates polyglutamine aggregation. Mol Cell Biol. 2008;28(17):5196-5208.

32. Fujita Y, Yamashita T. Axon growth inhibition by RhoA/ROCK in the central nervous system. Front Neurosci. 2014;8:338.

33. $\mathrm{Wu} \mathrm{CH}$, et al. Mutations in the profilin 1 gene cause familial amyotrophic lateral sclerosis. Nature. 2012;488(7412):499-503.

34. Kovar DR, Harris ES, Mahaffy R, Higgs HN, Pollard TD. Control of the assembly of ATPand ADP-actin by formins and profilin. Cell. 2006;124(2):423-435.

35. Kovar DR, Kuhn JR, Tichy AL, Pollard TD. The fission yeast cytokinesis formin Cdc12p is a barbed end actin filament capping protein gated by profilin. J Cell Biol. 2003;161(5):875-887.

36. Bartolini F, Moseley JB, Schmoranzer J, Cassimeris L, Goode BL, Gundersen GG. The formin $\mathrm{mDia} 2$ stabilizes microtubules independently of its actin nucleation activity. J Cell Biol. 2008;181(3):523-536.

37. Chesarone MA, DuPage AG, Goode BL. Unleashing formins to remodel the actin and microtubule cytoskeletons. Nat Rev Mol Cell Biol. 2010;11(1):62-74

38. Rizvi SA, et al. Identification and characterization of a small molecule inhibitor of formin-mediated actin assembly. Chem Biol.
2009;16(11):1158-1168.

39. Dent EW, Gupton SL, Gertler FB. The growth cone cytoskeleton in axon outgrowth and guidance. Cold Spring Harb Perspect Biol. 2011;3(3):a001800.

40. Prokop A, Beaven R, Qu Y, Sánchez-Soriano $\mathrm{N}$. Using fly genetics to dissect the cytoskeletal machinery of neurons during axonal growth and maintenance. JCell Sci. 2013;126(pt 11):2331-2341.

41. Qu Y, et al. Efa6 protects axons and regulates their growth and branching by inhibiting microtubule polymerisation at the cortex. Elife. 2019;8:e50319.

42. Chan KY, et al. Engineered AAVs for efficient noninvasive gene delivery to the central and peripheral nervous systems. Nat Neurosci. 2017;20(8):1172-1179.

43. Coles $\mathrm{CH}$, Bradke F. Coordinating neuronal actin-microtubule dynamics. Curr Biol. 2015;25(15):R677-R691.

44. Lee CW, Vitriol EA, Shim S, Wise AL, Velayutham RP, Zheng JQ. Dynamic localization of G-actin during membrane protrusion in neuronal motility. Curr Biol. 2013;23(12):1046-1056.

45. Dent EW, Gertler FB. Cytoskeletal dynamics and transport in growth cone motility and axon guidance. Neuron. 2003;40(2):209-227.

46. Lin CH, Forscher P. Growth cone advance is inversely proportional to retrograde $\mathrm{F}$-actin flow. Neuron. 1995;14(4):763-771.

47. Shimada T, et al. Shootin1 interacts with actin retrograde flow and L1-CAM to promote axon outgrowth. J Cell Biol. 2008;181(5):817-829.

48. Nichol RH, Hagen KM, Lumbard DC, Dent EW, Gómez TM. Guidance of axons by local coupling of retrograde flow to point contact adhesions. J Neurosci. 2016;36(7):2267-2282.

49. Schaefer AW, Kabir N, Forscher P. Filopodia and actin arcs guide the assembly and transport of two populations of microtubules with unique dynamic parameters in neuronal growth cones. JCell Biol. 2002;158(1):139-152.

50. Lin CH, Espreafico EM, Mooseker MS, Forscher P. Myosin drives retrograde F-actin flow in neuronal growth cones. Biol Bull. 1997;192(1):183-185.

51. Yvon AM, Gross DJ, Wadsworth P. Antagonistic forces generated by myosin II and cytoplasmic dynein regulate microtubule turnover, movement, and organization in interphase cells. Proc Natl Acad Sci U S A. 2001;98(15):8656-8661.

52. Myers KA, Tint I, Nadar CV, He Y, Black MM, Baas PW. Antagonistic forces generated by cytoplasmic dynein and myosin-II during growth cone turning and axonal retraction. Traffic. 2006;7(10):1333-1351.

53. Witke $\mathrm{W}$, et al. In mouse brain profilin I and profilin II associate with regulators of the endocytic pathway and actin assembly. EMBO J. 1998;17(4):967-976.

54. Da Silva JS, Medina M, Zuliani C, Di Nardo A, Witke W, Dotti CG. RhoA/ROCK regulation of neuritogenesis via profilin IIa-mediated control of actin stability. JCell Biol. 2003;162(7):1267-1279.

55. Henty-Ridilla JL, Rankova A, Eskin JA, Kenny K, Goode BL. Accelerated actin filament polymerization from microtubule plus ends. Science. 2016;352(6288):1004-1009.

56. Marx A, Godinez WJ, Tsimashchuk V, Bank- 


\section{RESEARCH ARTICLE}

head P, Rohr K, Engel U. Xenopus cytoplasmic linker-associated protein 1 (XCLASP1) promotes axon elongation and advance of pioneer microtubules. Mol Biol Cell. 2013;24(10):1544-1558.

57. Böttcher RT, et al. Profilin 1 is required for abscission during late cytokinesis of chondrocytes. ЕМBO J. 2009;28(8):1157-1169.

58. Young P, Qiu L, Wang D, Zhao S, Gross J, Feng G. Single-neuron labeling with inducible Cre-mediated knockout in transgenic mice. Nat Neurosci. 2008;11(6):721-728.

59. Leite SC, et al. The actin-binding protein $\alpha$-ad- ducin is required for maintaining axon diameter. Cell Rep. 2016;15(3):490-498.

60. Kaech S, Banker G. Culturing hippocampal neurons. Nat Protoc. 2006;1(5):2406-2415.

61. Schmitz SK, et al. Automated analysis of neuronal morphology, synapse number and synaptic recruitment. J Neurosci Methods. 2011;195(2):185-193.

62. Riedl J, et al. Lifeact: a versatile marker to visualize F-actin. Nat Methods. 2008;5(7):605-607.

63. Efimov A, et al. Paxillin-dependent stimulation of microtubule catastrophes at focal adhesion sites.
The Journal of Clinical Investigation

JCell Sci. 2008;121(pt 2):196-204.

64. Stepanova T, et al. Visualization of microtubule growth in cultured neurons via the use of EB3GFP (end-binding protein 3-green fluorescent protein). J Neurosci. 2003;23(7):2655-2664.

65. da Silva TF, et al. Peripheral nervous system plasmalogens regulate Schwann cell differentiation and myelination. JClin Invest. 2014;124(6):2560-2570.

66. Jockusch BM, Murk K, Rothkegel M. The profile of profilins. Rev Physiol Biochem Pharmacol. 2007;159:131-149. 